\title{
Unique continuation from Cauchy data in unknown non-smooth domains
}

\author{
LUCA RONDI
}

\begin{abstract}
We consider a conducting body which presents some (unknown) perfectly insulating defects, such as cracks or cavities, for instance. We perform measurements of current and voltage type on a (known) part of the boundary of the conductor. We prove that, even if the defects are unknown, the current and voltage measurements at the boundary uniquely determine the corresponding electrostatic potential inside the conductor. A corresponding stability result, related to the stability of Neumann problems with respect to domain variations, is also proved. Some applications of these results to inverse problems are presented.

Mathematics Subject Classification (2000): 35B60 (primary); 35J25, 35R30 (secondary).
\end{abstract}

\section{Introduction}

An electrically conducting body occupies a region $\Omega$, which we assume to be a bounded domain in $\mathbb{R}^{N}, N \geq 2$, with a reasonably smooth boundary. We suppose that the conductor is homogeneous and isotropic. Let us assume that such a conductor presents some perfectly insulating defects, such as cracks (either interior or surface breaking), cavities or material losses at the boundary, which might be caused by different phenomena, like for instance fractures or corrosion. The boundaries of these defects are collected into a closed set which we call $K$. If we prescribe a current density $f \in L^{2}(\partial \Omega)$, with $\int_{\partial \Omega} f=0$ and such that its support is contained in $\tilde{\gamma}$, a part of the boundary of $\Omega$ which is accessible, known and disjoint from $K$, then the electrostatic potential $u=u(f, K)$ inside the conductor solves the following Neumann-type boundary-value problem, whose precise formulation will be discussed in Section 3:

$$
\begin{cases}\frac{\Delta u=0}{\partial u}=f & \text { in } \Omega \backslash K, \\ \frac{\partial u}{\partial v}=0 & \text { on } \partial(\Omega \backslash K) \backslash \tilde{\gamma} .\end{cases}
$$

This work is supported by MIUR under grant n. 2004011204.

Received January 27, 2006; accepted in revised form May 16, 2006. 
The motivation of our work relies in the following kind of inverse problem. Assuming that the defect $K$ is unknown, we might wish to determine it by performing boundary measurements of voltage and current type. That is, we prescribe one or more currents $f$ and we measure on $\gamma$, an accessible and known part of $\partial \Omega$, the value of the corresponding potentials $u$. Through these measurements we obtain additional information with which we would like to recover the unknown defect $K$. For what concerns the determination of cracks we refer to the recent review paper [5], where uniqueness, stability and reconstruction procedures, in two and three dimensions, are discussed. For the determination of other defects, such as cavities or material losses at the boundary, we refer to the following papers and to the references therein. The uniqueness and stability issues are treated in [21], for the two-dimensional case, and in [1], for the higher-dimensional case.

A two-step procedure is usually employed to deal with these kinds of inverse problems, see for instance [11] and [3]. In the first step, the potential is recovered from the boundary measurements of voltage and current type. Subsequently, in the second step, features of the potential such as singularities, level sets or critical points are used to determine the unknown defect $K$. For instance, in our case, that is when the defects are perfectly insulating, the jump set $S(u)$ of $u$ is contained in $K$. Thus $S(u)$ would identify at least a part of our defect. Repeating the procedure for different and suitable choices of $f$, the union of the jump sets of the corresponding potentials would cover the whole $K$. The uniqueness results which are available in the literature give us information on how many and which kind of measurements we need to take in order to identify uniquely, at least in a suitable class of admissible defects, the unknown $K$. Here we limit ourselves to notice that in many interesting cases a finite number (usually one or two) of suitably chosen measurements is enough. However, in dimesion higher than 2, for what concerns insulating cracks, still a general uniqueness result with a finite number of measurements is missing, as the only available result, [3], deals with planar cracks only.

In this paper we investigate the first step of the previous scheme. Let us suppose that $K$ is the unknown defect. Fixed a current density $f$, we measure $g=$ $\left.u(f, K)\right|_{\gamma}$, where $u(f, K)$ solves (1.1). Given $f$ and $g$, we ask whether the potential inside the conductor is uniquely identified by the Cauchy data $(g, f)$. In other words, we ask when the following result holds: whenever another defect $K_{1}$ gives rise to the same boundary measurement $g$, that is we have $\left.u(f, K)\right|_{\gamma}=\left.u\left(f, K_{1}\right)\right|_{\gamma}$, then $u(f, K)=u\left(f, K_{1}\right)$ in $\Omega$. Such a unique continuation result is relatively easy to prove if we assume $K$ and $K_{1}$ smooth. However, when the unknown defect $K$ is very irregular, various technical difficulties arise. The aim of our work is to show that uniqueness holds for a very large class of admissible defects.

The main result is the following. When $K$ is formed, up to a set of zero capacity, by Lipschitz hypersurfaces and $K_{1}$ has finite $(N-1)$-dimensional Hausdorff measure, then $\left.u(f, K)\right|_{\gamma}=\left.u\left(f, K_{1}\right)\right|_{\gamma}$ implies $u(f, K)=u\left(f, K_{1}\right)$ in $\Omega$, see Theorem 3.3. The proof is strongly based on harmonic analysis techniques on Lipschitz domains, in particular for what concerns the Neumann problem. In fact, see [15], a Lipschitz condition on the boundary allows us to pass from a weak formulation of the Neumann datum to a pointwise formulation, through the nontangential max- 
imal function and the nontangential limit. This procedure allows us to deal with the weak smoothness of $K$. In order to deal with the roughness of $K_{1}$, we use another key ingredient, namely the Gauss-Green formula for sets of finite perimeter. Furthermore, in two dimensions, we may take advantage of the duality provided by the use of harmonic conjugates, thus other kinds of unique continuation results may be proved, see Theorem 3.4.

As a first application of our unique continuation results to inverse problems, we deduce uniqueness with a single measurement for the inverse problem of determining cavities or material losses at the boundary, see Theorem 3.6. On the other hand it is well known that a single measurement is not enough, in general, to determine cracks, see for instance [11].

In order to implement the reconstruction procedure of (a part of) $K$ from the Cauchy data $(g, f)$, through the determination of the potential $u=u(f, K)$ and of its jump set $S(u)$, we might set the problem into the following least-square formulation. We look for

$$
\min _{\tilde{K}}\left\|\left.u(f, \tilde{K})\right|_{\gamma}-g\right\|_{L^{2}(\gamma)}
$$

where $\tilde{K}$ varies in a suitable class of admissible defects. The minimum is zero and is obviously reached for $\tilde{K}=K$. Although other $\tilde{K}$ may be minimizers, the previous uniqueness results imply that for any minimizer $\tilde{K}$ we have $u(f, \tilde{K})=$ $u(f, K)$. However, one usually has to deal with noisy measurements, that is $f$, the prescribed current density, and in particular $g$, the measured potential at the boundary, are known up to some noise which is due to the errors the measurements are subject to. Hence, we need to investigate the stability of the unique continuation results described before. We require at least a qualitative type of stability, that is convergence in a suitable sense to the looked-for potential when the noise goes to zero. We prove two kinds of, strictly related, stability results, under minimal assumptions of regularity on the admissible defects, whose main features are that we treat the three dimensional case and that the admissible defects may include cracks.

First, in Theorem 6.2, we show stability of the direct problem (1.1) under the variation of $K$, with respect to the Hausdorff distance, which corresponds to the stability of Neumann problem under domain variations. We refer to [6] and to [12], and their references, for a detailed account on this problem, in two dimensions and in dimensions higher than 2, respectively. Although our approach and proof are different, our result is quite similar to the one obtained in [12]. On the other hand, in dimension 2, again by making use of duality arguments, stronger results have been proved, see again [6] and its references.

Then, in Theorem 6.3, we prove stability of the unique continuation in $\Omega$ of $u$ from the Cauchy data. As an application to inverse problems of Theorems 6.2 and 6.3, we show in Theorem 6.6 that, in the class of admissible defects for which stability holds, the least-square problem (1.2) is stable with respect to noise on the Cauchy data. 
We observe that the difficulty of proving such stability results is due to the illposedness of these unique continuations from the Cauchy data. We remark that the ill-posedness of the inverse boundary-value problems we are considering is strictly linked to the one of the unique continuation problem. We recall that, however, under stronger assumptions on the admissible defects, quantitative stability results for the inverse problems have been obtained, see for instance [21] and, for what concerns cracks, [22] for the two-dimensional case, and [1] for cavities and material losses in higher dimensions. Although such quantitative estimates of stability are very weak, indeed of logarithmic type, they are essentially optimal, since these kinds of inverse problems are severely ill-posed, as shown for instance in [8].

An interesting prosecution of this work would be to develop a numerical procedure for the determination of the potential, and in particular of its jump set, from the Cauchy data, by variational methods, for instance in $S B V(\Omega)$, the space of special functions of bounded variation to which the potential $u$ belongs. Such a numerical method should take into account the noise of the data, the conditions under which stability occurs and the fact that the jump sets of $S B V$ functions are quite difficult to handle from a numerical point of view. For example, a quite natural attempt would be to use a modification of the so-called Mumford-Shah functional which has been introduced in $[18,19]$ as an image segmentation method. This paper may be also seen as a first step in this direction. However such an issue requires further investigation and it will be the subject of future research. We also recall that the Mumford-Shah functional has been already used, from a different point of view, in the context of inverse problems as a regularization term for the determination of discontinuous conductivities, see [23].

The plan of the paper is as follows. In Section 2 we recall some arguments of geometric measure theory which will be used in the sequel. In Section 3, first we consider problem (1.1) and prove some properties of its solution. Then we state the main results of unique continuation, Theorem 3.3 and Theorem 3.4, and discuss the applications to inverse problems, Theorem 3.6. Section 4 and Section 5 are devoted to the proofs of Theorem 3.3 and Theorem 3.4, respectively. Finally, Section 6 contains the stability results, Theorem 6.2 and Theorem 6.3, their proofs and their applications to inverse problems, Theorem 6.6.

\section{Preliminaries}

Throughout the paper the integer $N \geq 2$ will denote the dimension of the space. For every $x \in \mathbb{R}^{N}$, we shall set $x=\left(x^{\prime}, x_{N}\right)$, where $x^{\prime} \in \mathbb{R}^{N-1}$ and $x_{N} \in \mathbb{R}$, and, for any $r>0$, we shall denote by $B_{r}(x)$ and $B_{r}^{\prime}\left(x^{\prime}\right)$, respectively, the open ball in $\mathbb{R}^{N}$ centred at $x$ of radius $r$ and the open ball in $\mathbb{R}^{N-1}$ centred at $x^{\prime}$ of radius $r$. Usually we shall write $B_{r}$ and $B_{r}^{\prime}$ instead of $B_{r}(0)$ and $B_{r}^{\prime}(0)$, respectively. Furthermore, for any $r>0$ and $t>0$, we set $Q_{r}^{\prime}\left(x^{\prime}\right)=\prod_{i=1}^{N-1}\left(x_{i}-r, x_{i}+r\right) \subset \mathbb{R}^{N-1}$ and $Q_{r, t}(x)=\left\{y=\left(y^{\prime}, y_{N}\right) \in \mathbb{R}^{N}: y^{\prime} \in Q_{r}^{\prime}\left(x^{\prime}\right), y_{N} \in\left(x_{N}-t, x_{N}+t\right)\right\}$. Again, $Q_{r}^{\prime}$ and $Q_{r, t}$ shall denote $Q_{r}^{\prime}(0)$ and $Q_{r, t}(0)$, respectively. 
For any non negative integer $k$ we denote by $\mathcal{H}^{k}$ the $k$-dimensional Hausdorff measure, see [10] for a definition and for its main properties. We recall that for Borel subsets of $\mathbb{R}^{N}$ the $N$-dimensional Hausdorff measure coincides with $\mathcal{L}^{N}$, the $N$-dimensional Lebesgue measure. Furthermore, if $\gamma \subset \mathbb{R}^{N}$ is a smooth manifold of dimension $k$, then $\mathcal{H}^{k}$ restricted to $\gamma$ coincides with its $k$-dimensional surface measure. For any Borel $E \subset \mathbb{R}^{N}$ we let $|E|=\mathcal{L}^{N}(E)$ and $[E]=\mathcal{H}^{N-1}(E)$.

We recall that a bounded domain $\Omega \in \mathbb{R}^{N}$ is said to have a Lipschitz boundary if for every $x \in \partial \Omega$ there exist a Lipschitz function $\varphi: \mathbb{R}^{N-1} \rightarrow \mathbb{R}$ and a positive constant $r$ such that for any $y \in B_{r}(x)$ we have, up to a rigid transformation,

$$
y \in \Omega \quad \text { if and only if } y_{N}<\varphi\left(y^{\prime}\right) .
$$

We say that a function $\varphi: A \rightarrow B, A$ and $B$ being metric spaces, is bi-Lipschitz if it is invertible and $\varphi$ and $\varphi^{-1}: \varphi(A) \rightarrow A$ are both Lipschitz functions. If both the Lipschitz constants of $\varphi$ and $\varphi^{-1}$ are bounded by $L>0$, then we say that $\varphi$ is bi-Lipschitz with constant $L$.

We also recall some basic notation and properties of functions of bounded variation and sets of finite perimeter. For a more comprehensive treatment of these subjects see, for instance, [4, 9, 10, 13].

Given an open bounded set $\Omega \subset \mathbb{R}^{N}$, we denote by $B V(\Omega)$ the Banach space of functions of bounded variation. We recall that $u \in B V(\Omega)$ if and only if $u \in$ $L^{1}(\Omega)$ and its distributional derivative $D u$ is a bounded vector measure. We endow $B V(\Omega)$ with the standard norm as follows. Given $u \in B V(\Omega)$, we denote by $|D u|$ the total variation of its distributional derivative and we set $\|u\|_{B V(\Omega)}=\|u\|_{L^{1}(\Omega)}+$ $|D u|(\Omega)$. We say that a sequence of $B V(\Omega)$ functions $\left\{u_{h}\right\}_{h=1}^{\infty}$ converges weakly* in $B V(\Omega)$ if and only if $u_{h}$ converges to $u$ in $L^{1}(\Omega)$ and $D u_{h}$ weakly* converges to $D u$ in $\Omega$, that is

$$
\lim _{h \rightarrow \infty} \int_{\Omega} v \mathrm{~d} D u_{h}=\int_{\Omega} v \mathrm{~d} D u \quad \text { for any } v \in C_{0}(\Omega) .
$$

We denote by $S B V(\Omega)$ the space of special functions of bounded variation that is the space of functions $u \in B V(\Omega)$ so that $D u$ has a singular part, with respect to the $N$-dimensional Lebesgue measure, concentrated on $S(u), S(u)$ being the approximate discontinuity set (or jump set) of $u$. The density of the absolutely continuous part of $D u$ with respect to the $N$-dimensional Lebesgue measure will be denoted by $\nabla u$, the approximate gradient of $u$.

The special functions of bounded variation satisfy the following compactness and semicontinuity theorem.

Theorem 2.1 (SBV Compactness and Semicontinuity). For any fixed $p, 1<p<$ $+\infty$, if $\left\{u_{h}\right\}_{h=1}^{\infty}$ is a sequence of functions belonging to $S B V(\Omega)$ satisfying for a given constant $C>0$

$$
\left\|u_{h}\right\|_{B V(\Omega)} \leq C, \quad \text { for any } h
$$


and

$$
\int_{\Omega}\left|\nabla u_{h}\right|^{p}+\left[S\left(u_{h}\right)\right] \leq C, \quad \text { for any } h,
$$

then we may extract a subsequence, which we relabel $\left\{u_{k}\right\}_{k=1}^{\infty}$, such that $u_{k}$ converges weakly* in $B V(\Omega)$ to a function $u \in S B V(\Omega)$ and the following lower semicontinuity properties hold

$$
[S(u)] \leq \liminf _{k}\left[S\left(u_{k}\right)\right] ; \quad \int_{\Omega}|\nabla u|^{p} \leq \liminf _{k} \int_{\Omega}\left|\nabla u_{k}\right|^{p} .
$$

Proof. See for instance [4, Theorem 4.7 and Theorem 4.8].

Remark 2.2. In the previous theorem the uniform $B V(\Omega)$ norm bound (2.2) can be replaced by the following uniform $L^{\infty}(\Omega)$ norm bound

$$
\left\|u_{h}\right\|_{L^{\infty}(\Omega)} \leq C, \quad \text { for any } h .
$$

The following remark can also be useful. Let $u \in B V(\Omega)$. If $a, b$ are two real numbers so that $a<b$ and we denote $v=(u \wedge b) \vee a=\max \{\min \{u, b\}, a\}$, then $v \in B V(\Omega)$ and

$$
|\nabla v| \leq|\nabla u| \quad \text { a.e. in } \Omega ; \quad[S(v) \backslash S(u)]=0 ; \quad|D v|(\Omega) \leq|D u|(\Omega) .
$$

Note that if $u \in S B V(\Omega)$ then also $v \in S B V(\Omega)$.

Let $E$ be a bounded Borel set contained in $\mathbb{R}^{N}$ and let $r>0$ be such that $E$ is compactly contained in $B_{r}$. We say that $E$ is a set of finite perimeter if its characteristic function $\chi_{E}$ belongs to $B V\left(B_{r}\right)$ and we call the number $P(E)=$ $\left|D \chi_{E}\right|\left(B_{r}\right)$ its perimeter.

For any set $E$ of finite perimeter, let $\partial^{*} E$ be the reduced boundary in the De Giorgi sense, that is the set of $x \in \mathbb{R}^{N}$ such that $\left|D \chi_{E}\right|\left(B_{\rho}(x)\right)>0$ for any $\rho>0$ and there exists $v(x)$ with $|v(x)|=1$ such that

$$
\lim _{\rho \rightarrow 0^{+}} \frac{D \chi_{E}\left(B_{\rho}(x)\right)}{\left|D \chi_{E}\right|\left(B_{\rho}(x)\right)}=-v(x) .
$$

We call the function $v: \partial^{*} E \rightarrow \mathbb{S}^{N-1}$ the exterior normal to $E$. Let us also note that $\partial^{*} E \subset \partial \tilde{E}$ for any $\tilde{E}$ such that $\chi_{\tilde{E}}=\chi_{E}$ almost everywhere.

The following Gauss-Green formula holds true for sets of finite perimeter.

Theorem 2.3. Let $E$ be a bounded Borel set of finite perimeter. Then $\partial^{*} E$ is $\mathcal{H}^{N-1}$ measurable with $\left[\partial^{*} E\right]$ finite and

$$
\int_{E} \operatorname{div}(f)=\int_{\partial^{*} E} f \cdot v \mathrm{~d} \mathcal{H}^{N-1} \quad \text { for any } f \in C_{0}^{1}\left(\mathbb{R}^{N}, \mathbb{R}^{N}\right) .
$$

Proof. See for instance [10, Theorem 4.5.6] or [9, Section 5.8, Theorem 1]. 
Let us further remark that the intersection of two sets of finite perimeter is still a set of finite perimeter. Moreover, whenever $E$ is open and $[\partial E$ ] is finite, then $E$ is a set of finite perimeter, see for instance [9, Section 5.11, Theorem 1].

We shall make use of the notion of capacity. For any bounded domain $\Omega \subset \mathbb{R}^{N}$ and any $E \subset \Omega$ we define the capacity of the condenser $(E, \Omega)$ as

$$
\operatorname{cap}(E, \Omega)=\inf _{u \in S(E, \Omega)} \int_{\Omega}|\nabla u|^{2}
$$

where

$$
S(E, \Omega)=\left\{u \in H_{0}^{1}(\Omega): u=1 \text { a.e. in an open set containing } E\right\} .
$$

If $S(E, \Omega)$ is empty we set $\operatorname{cap}(E, \Omega)=\infty$. We remark that if $K$ is a compact set contained in $\Omega$, then we have

$$
\operatorname{cap}(K, \Omega)=\inf _{u \in S(E, \Omega) \cap C_{0}^{\infty}(\Omega)} \int_{\Omega}|\nabla u|^{2} .
$$

For basic properties of the capacity of condensers we refer to [14, Chapter 2]. Here we simply recall that the capacity is countably subadditive, that is if $\left\{E_{i}\right\}_{i=1}^{\infty}$ is a sequence of sets contained in $\Omega$ and $E=\bigcup_{i=1}^{\infty} E_{i}$, then

$$
\operatorname{cap}(E, \Omega) \leq \sum_{i=1}^{\infty} \operatorname{cap}\left(E_{i}, \Omega\right)
$$

We say that $E \subset \mathbb{R}^{N}$ has zero capacity if $\operatorname{cap}(E \cap \Omega, \Omega)=0$ for any $\Omega$ open and bounded subset of $\mathbb{R}^{N}$. By the subadditivity, a countable union of sets with zero capacity is still a set with zero capacity. Furthermore, $E$, a bounded subset of $\mathbb{R}^{N}$, has zero capacity if and only if there exists an open, bounded neighbourhood of $E$ such that $\operatorname{cap}(E, \Omega)=0$.

We have that the image of a bounded subset with zero capacity through a biLipschitz function is still a set with zero capacity. The following relationships hold between the Hausdorff measure and capacity. Let $E \subset \mathbb{R}^{N}$ be such that $\mathcal{H}^{N-2}(E)<\infty$, then $E$ has zero capacity. On the other hand, if $E \subset \mathbb{R}^{N}$ has zero capacity, then $\mathcal{H}^{N-2+\varepsilon}(E)=0$ for any $\varepsilon>0$.

\section{Uniqueness results}

Let $\Omega$ be a bounded domain contained in $\mathbb{R}^{N}, N \geq 2$, with Lipschitz boundary $\partial \Omega$. We observe that $[\partial \Omega]<+\infty$. Let us fix a number $p \geq 2$ such that $p>N-1$. For $N=2, p$ can be clearly chosen to be equal to 2 . We assume that $\Omega$ and $p$ are fixed throughout the paper.

Let us fix $\gamma$, a nonempty open subset of $\partial \Omega$, and $f \in L^{p}(\partial \Omega)$ such that $\int_{\partial \Omega} f=0$ and $f \not \equiv 0$. 
Let $K$ be an admissible defect, that is $K$ is a compact set contained in $\bar{\Omega}$ satisfying the following compatibility condition. We require that there exists a domain $\tilde{\Omega}_{1} \subset \Omega$ such that $\bar{\gamma} \cup \operatorname{supp}(f)$ is contained in the interior of $\partial \Omega \cap \partial \tilde{\Omega}_{1}$, and $\operatorname{dist}\left(K, \bar{\Omega}_{1}\right)>0$. Without loss of generality, we can assume that $\tilde{\Omega}_{1}$ has a Lipschitz boundary and that we can find another domain $\Omega_{1} \subset \tilde{\Omega}_{1}$ such that $\Omega_{1}$ has a Lipschitz boundary, $\bar{\gamma} \cup \operatorname{supp}(f)$ is contained in the interior of $\partial \Omega \cap \partial \Omega_{1}$ and $\operatorname{dist}\left(\bar{\Omega}_{1}, \partial \tilde{\Omega}_{1} \cap \Omega\right)>0$. We denote with $G_{K}$ the connected component of $\Omega \backslash K$ such that $\operatorname{supp}(f) \subset \partial G_{K}$. By the compatibility condition, we have that $\tilde{\Omega}_{1}$ is contained in $G_{K}$, therefore $\gamma \subset \partial G_{K}$ as well. Let us call, for the time being, $\tilde{\gamma}$ the support of $f$. Then, the geometric configuration is illustrated in Figure 1. We observe that the grey-coloured parts correspond to the connected components of $\Omega \backslash K$ which are different from $G_{K}$.

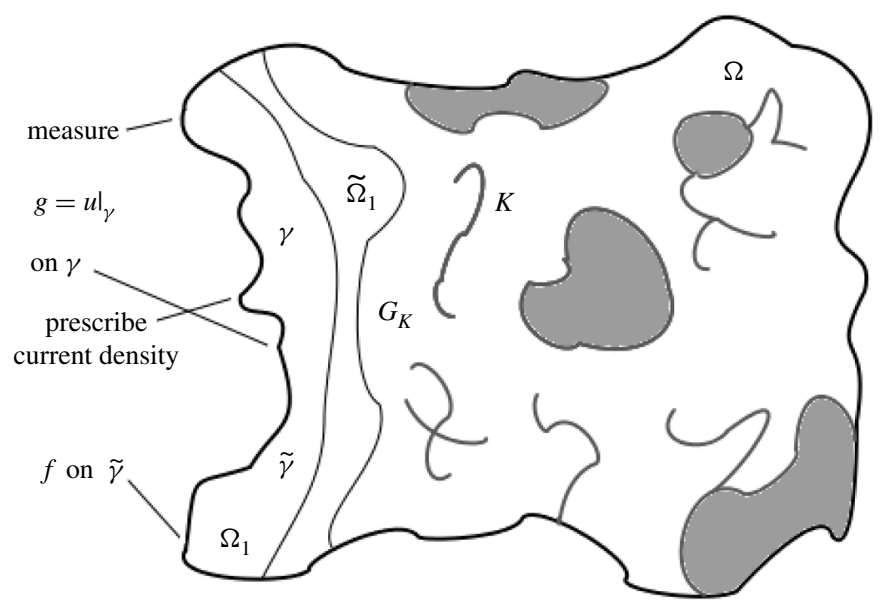

Figure 1. Geometric configuration.

The domain $\tilde{\Omega}_{1}$ can be seen, from a practical point of view, as a part of the body which is known to be safe (the defects $K$ do not intersect it) and whose exterior boundary is accessible, therefore we can prescribe current densities and perform voltage measurements there. From a technical point of view, we require some distance between the region where the current density is different from zero and Dirichlet data are available and the one where $K$ lies. The use of the domain $\Omega_{1}$ and the fact that the support of $f$ and $\gamma$ are compactly contained in the interior of $\partial \Omega \cap \partial \Omega_{1}$ are due to technical reasons, for instance they allow us to prove some regularity estimates upon $u$, the solution to (1.1), which depend on $K$ only through the domains $\Omega_{1}, \tilde{\Omega}_{1}$, the support of $f$ and $\gamma$, see Proposition 3.1. 
For any bounded open set $D \subset \mathbb{R}^{N}$, we set $L^{1,2}(D)$ as the following DenyLions space

$$
L^{1,2}(D)=\left\{u \in L_{\text {loc }}^{2}(D): \nabla u \in L^{2}\left(D, \mathbb{R}^{N}\right)\right\}
$$

For basic properties of Deny-Lions spaces we refer to [7] and [16]. As a convention, we identify two elements $u_{1}$ and $u_{2}$ of $L^{1,2}(D)$ whenever $\nabla u_{1}=\nabla u_{2}$ almost everywhere in $D$. We point out that if $D$ is bounded with Lipschitz boundary then any $v \in L^{1,2}(D)$ belongs to $H^{1}(D)$ and, obviously, viceversa. Finally, we notice that the set $\left\{\nabla u: u \in L^{1,2}(D)\right\}$ is a closed subspace of $L^{2}\left(D, \mathbb{R}^{N}\right)$.

Let $K$ be an admissible defect, then there exists a function $u=u(f, K) \in$ $L^{1,2}(\Omega \backslash K)$ such that

$$
\int_{\Omega \backslash K} \nabla u \cdot \nabla v=\int_{\partial \tilde{\Omega}_{1} \cap \partial \Omega} f v \quad \text { for every } v \in L^{1,2}(\Omega \backslash K) .
$$

Such a function is unique in the sense that the gradients of any two solutions to (3.2) coincide almost everywhere in $\Omega \backslash K$. We always take as $u$ the solution satisfying the following two normalization conditions. First,

$$
\int_{\gamma} u=0
$$

and, second, since $u$ is constant on any connected component of $\Omega \backslash K$ different from $G_{K}$, we pose

$$
u=0 \quad \text { almost everywhere in } \Omega \backslash G_{K} .
$$

In such a way, $u$ is defined almost everywhere in $\Omega$ and is the unique solution of (3.2)-(3.3)-(3.4).

We wish to remark that (3.2) is the weak formulation of the following Neumann type boundary-value problem

$$
\left\{\begin{array}{l}
\Delta u=0 \text { in } \Omega \backslash K \\
\frac{\partial u}{\partial v}=f \text { on } \partial \tilde{\Omega}_{1} \cap \partial \Omega \\
\frac{\partial u}{\partial v}=0 \text { on } \partial(\Omega \backslash K) \backslash\left(\partial \tilde{\Omega}_{1} \cap \partial \Omega\right) .
\end{array}\right.
$$

Here $\Omega \backslash K$ might represent an electrostatic conductor in which some perfectly insulating defects, given by $K$, are present. In such a case $u$ represents the electrostatic potential if the current density $f$ is applied on the boundary of the conductor. The electrostatic potential $u=u(f, K)$ strongly depends on $K$, apart from clearly depending on $\Omega$ and $f$.

In the next proposition, we state some basic properties of $u$. 
Proposition 3.1. Under the previous assumptions, let $K$ be an admissible defect and let $u$ be the solution to (3.2)-(3.3)-(3.4).

Then there exists a constant $C_{1}>0$, depending on $p, \Omega, \gamma, \operatorname{supp}(f)$ and $K$ only, such that

$$
\begin{gathered}
\|\nabla u\|_{L^{2}(\Omega \backslash K)} \leq C_{1}\|f\|_{L^{2}(\partial \Omega)}, \\
\|u\|_{L^{\infty}(\Omega)} \leq C_{1}\|f\|_{L^{p}(\partial \Omega)} .
\end{gathered}
$$

Proof. We begin with the following observation. We have that there exists a constant $C_{2}$, depending on $\tilde{\Omega}_{1}, \Omega$ and $\gamma$ only, such that the following Poincare type inequality holds

$\int_{\tilde{\Omega}_{1}} v^{2}+\int_{\partial \tilde{\Omega}_{1} \cap \partial \Omega} v^{2} \leq C_{2} \int_{\tilde{\Omega}_{1}}|\nabla v|^{2}$ for any $v \in H^{1}\left(\tilde{\Omega}_{1}\right)$ such that $\int_{\gamma} v=0$.

Then, by (3.2), we infer that

$$
\int_{\Omega \backslash K}|\nabla u|^{2} \leq\|f\|_{L^{2}\left(\partial \tilde{\Omega}_{1} \cap \partial \Omega\right)}\|u\|_{L^{2}\left(\partial \tilde{\Omega}_{1} \cap \partial \Omega\right)} .
$$

Hence (3.6) immediately follows from (3.8).

Next, we show that the following estimate holds

$$
\|u\|_{L^{\infty}\left(\bar{\Omega}_{1}\right)} \leq C_{3}\|f\|_{L^{p}(\partial \Omega)},
$$

where $C_{3}$ depends on $\Omega_{1}, \tilde{\Omega}_{1}, \Omega, \gamma$ and $p$ only.

We denote by $\tilde{u} \in H^{1}\left(\tilde{\Omega}_{1}\right)$ the weak solution to the following boundary-value problem

$$
\left\{\begin{array}{l}
\Delta \tilde{u}=0 \text { in } \tilde{\Omega}_{1}, \\
\frac{\partial \tilde{u}}{\partial v}=f \text { on } \partial \tilde{\Omega}_{1} \cap \partial \Omega \\
\frac{\partial \tilde{u}}{\partial v}=0 \text { on } \partial \tilde{\Omega}_{1} \cap \Omega,
\end{array}\right.
$$

with the normalization condition

$$
\int_{\gamma} \tilde{u}=0
$$

By standard global regularity estimates, for instance by a simple modification of arguments in [20], we can show that there exists $C_{4}$, depending on $\tilde{\Omega}_{1}, \gamma$ and $p$ only, such that $\tilde{u}$, solution to (3.10)-(3.11), satisfies

$$
\|\tilde{u}\|_{L^{\infty}\left(\tilde{\Omega}_{1}\right)} \leq C_{4}\|f\|_{L^{p}(\partial \Omega)}
$$


The function $\hat{u}=u-\tilde{u}$ satisfies

$$
\left\{\begin{array}{l}
\Delta \hat{u}=0 \text { in } \tilde{\Omega}_{1}, \\
\frac{\partial \hat{u}}{\partial \nu}=0 \text { on } \partial \tilde{\Omega}_{1} \cap \partial \Omega,
\end{array}\right.
$$

and furthermore, by the weak formulation of (3.10), by (3.8) and (3.6), we have that

$$
\|\hat{u}\|_{H^{1}\left(\tilde{\Omega}_{1}\right)} \leq C_{5}\|f\|_{L^{2}(\partial \Omega)},
$$

where $C_{5}$ depends on $\tilde{\Omega}_{1}, \Omega$ and $\gamma$ only. Standard regularity estimates up to the boundary imply that there exist $\beta_{1}, 0<\beta_{1}<1$, and $C_{6}$, depending on $\Omega_{1}, \tilde{\Omega}_{1}, \Omega$ and $\gamma$ only, such that

$$
\|\hat{u}\|_{C^{\beta_{1}\left(\bar{\Omega}_{1}\right)}} \leq C_{6}\|f\|_{L^{2}(\partial \Omega)} .
$$

Hence, estimate (3.9) immediately follows from (3.12) and (3.13).

Finally, we show that (3.7) is a consequence of (3.9). In fact, we have that $u$ is the unique solution (up to equivalence in $L^{1,2}(\Omega \backslash K)$ ) of the following problem

$$
\min _{\substack{\left.v \in L^{1,2}(\Omega \backslash K) \\ v\right|_{\Omega_{1}}=\left.u\right|_{\Omega_{1}}}} \int_{\Omega \backslash K}|\nabla v|^{2} .
$$

So, by a truncation argument, we can infer that $\|u\|_{L^{\infty}(\Omega \backslash K)} \leq\|u\|_{L^{\infty}\left(\Omega_{1}\right)}$ and the proof is concluded.

We wish to notice that the constant $C_{1}$ in the previous proposition depends on $K$ and $\operatorname{supp}(f)$ only via the two sets $\Omega_{1}$ and $\tilde{\Omega}_{1}$.

We remark that, in view of (3.7), $u$ actually belongs to $H^{1}(\Omega \backslash K)$. Furthermore, under the additional assumption that $[K]<+\infty$, or equivalently that $\left[\partial G_{K}\right]<$ $+\infty$, we have that $u$ belongs to $S B V(\Omega)$, its approximate discontinuity set $S(u)$ satisfies $\left[S(u) \backslash \partial G_{K}\right]=0$ and, finally, $\nabla u$, the weak derivative of $u$ in $\Omega \backslash K$, coincides almost everywhere in $\Omega$ with the approximate gradient of $u$, see for instance [4, Proposition 4.4].

Our aim is to recover the potential $u$ through the measurement of its value on $\gamma$. The first question that arises in a natural way is the following: given $f$, does $g=\left.u\right|_{\gamma}$ determine uniquely the potential $u$ ? In other words, we need a unique continuation property from the Cauchy data $(g, f)$ for harmonic functions inside a conductor with (unknown) insulating defects.

In order to obtain such a unique continuation result, we need to restrict our attention to a class of admissible defects satisfying a minimal regularity assumption.

Definition 3.2. Let $\mathcal{A}$ be the set of all admissible defects $K$ satisfying the following property.

There exists a closed set $\kappa \subset \partial G_{K}$ with zero capacity such that for every $x \in \partial G_{K} \backslash \kappa$ there exist a Lipschitz function $\varphi: \mathbb{R}^{N-1} \rightarrow \mathbb{R}$ and a positive constant $r$ such that we have, up to a rigid transformation,

$$
\partial G_{K} \cap B_{r}(x)=\left\{y=\left(y^{\prime}, y_{N}\right) \in B_{r}(x): y_{N}=\varphi\left(y^{\prime}\right)\right\} .
$$


By our assumptions on $\partial \Omega$, we immediately infer that the empty set belongs to $\mathcal{A}$. As an example of $K$ belonging to $\mathcal{A}$, we may think of $K$ such that $\partial G_{K}$ is composed by the finite union of sets $\gamma_{i}, i=1, \ldots, n$, where each $\gamma_{i}$ is a Lipschitz $(N-1)$-manifold whose boundary has zero capacity (for instance the boundary of $\gamma_{i}$ is an $(N-2)$-manifold) and the $\gamma_{i}$ are pairwise internally disjoint. In such a way we may model the simultaneous presence of defects such as cracks, cavities, surface breaking cracks and material losses at the boundary.

The following global unique continuation property provides the unique determination of the potential by measuring it on a suitable part of the boundary.

Theorem 3.3. Let $K_{1}$ belong to $\mathcal{A}$ and $K_{2}$ be an admissible defect such that $\left[\partial G_{K_{2}}\right]$ is finite.

Let $u_{1}=u\left(f, K_{1}\right)$ and $u_{2}=u\left(f, K_{2}\right)$ be the solutions to (3.2)-(3.3)-(3.4) with $K$ replaced by $K_{1}$ and $K_{2}$, respectively. If $u_{1}=u_{2}$ in the trace sense on $\gamma$, then $u_{1}=u_{2}$ almost everywhere in $\Omega$.

In two dimensions, other unique continuation results of this kind may be obtained. Let us consider $N=2$ and let us assume that $\operatorname{supp}(f) \subset \tilde{\gamma}_{0}$, where $\tilde{\gamma}_{0}$ is an open connected subset of $\partial \Omega$, that is an open subarc of $\partial \Omega$. We say that an admissible defect $K$ is compatible with $\tilde{\gamma}_{0}$ if $\tilde{\gamma}_{0} \subset \partial \tilde{\Omega}_{1} \cap \partial \Omega$.

We recall that a continuum is a connected set which is not reduced to a single point. Let $\mathcal{B}$ be the set of admissible defects $K$ compatible with $\tilde{\gamma}_{0}$ such that $\partial G_{K}=$ $\bigcup_{i=0}^{n} \gamma_{i}$, where $\gamma_{i}, i=0,1, \ldots, n$, are pairwise disjoint closed continua. Then the following result holds.

Theorem 3.4. Let $N=2$ and let us assume that $\operatorname{supp}(f) \subset \tilde{\gamma}_{0}$, where $\tilde{\gamma}_{0}$ is an open connected subset of $\partial \Omega$. Let $K_{1}$ belong to $\mathcal{B}$ and $K_{2}$ be an admissible defect such that either $K_{2}$ belongs to $\mathcal{B}$ or $\left[\partial G_{K_{2}}\right]$ is finite.

Let $u_{1}=u\left(f, K_{1}\right)$ and $u_{2}=u\left(f, K_{2}\right)$ be the solutions to (3.2)-(3.3)-(3.4) with $K$ replaced by $K_{1}$ and $K_{2}$, respectively. If $u_{1}=u_{2}$ in the trace sense on $\gamma$, then $u_{1}=u_{2}$ almost everywhere in $\Omega$.

We postpone the proof of Theorem 3.3 to Section 4 and the proof of Theorem 3.4 to Section 5. We conclude this section with the applications to inverse problems. We begin with the following corollary.

Corollary 3.5. Under the same assumptions either of Theorem 3.3 or of Theorem 3.4, if $u_{1}=u_{2}$ in the trace sense on $\gamma$, then the symmetric difference between $G_{K_{1}}$ and $G_{K_{2}}$ has Lebesgue measure zero.

Proof. It follows immediately from the fact that any harmonic function which is zero on a set of positive Lebesgue measure must be zero.

We remark that we do not obtain that $G_{K_{1}}$ and $G_{K_{2}}$ are equal, we only obtain that their reduced boundaries are equal. Therefore, they may differ for instance by the presence of some cracks. In order to have that $\left.u\right|_{\gamma}$ uniquely identifies $G_{K}$, we need to impose further restrictions on $G_{K}$. For example, let $\mathcal{A}^{\prime}$ be the class of 
admissible defects $K$ such that $K \in \mathcal{A},\left[\partial G_{K}\right]$ is finite and $G_{K}$ is the interior set of its own closure. In practice, we require that $K \in \mathcal{A}^{\prime}$ does not contain any crack and is formed by cavities and material losses at the boundary, only. Analogously, for $N=2$ and when $\operatorname{supp}(f) \subset \tilde{\gamma}_{0}$, where $\tilde{\gamma}_{0}$ is an open connected subset of $\partial \Omega$, we say that $K \in \mathcal{B}^{\prime}$ if $K \in \mathcal{B}$ and $G_{K}$ is the interior set of its own closure. Then we have the following uniqueness result, which is an immediate consequence of Corollary 3.5 .

Theorem 3.6. Let $K_{1}$ and $K_{2}$ either belong to $\mathcal{A}^{\prime}$ or, if $N=2$ and $\operatorname{supp}(f) \subset$ $\tilde{\gamma}_{0}$, where $\tilde{\gamma}_{0}$ is an open connected subset of $\partial \Omega$, to $\mathcal{B}^{\prime}$. Let $u_{1}=u\left(f, K_{1}\right)$ and $u_{2}=u\left(f, K_{2}\right)$ be the solutions to (3.2)-(3.3)-(3.4) with $K$ replaced by $K_{1}$ and $K_{2}$, respectively. If $u_{1}=u_{2}$ in the trace sense on $\gamma$, then $G_{K_{1}}=G_{K_{2}}$.

\section{Proof of Theorem 3.3}

We observe that we can find two domains $\Omega_{1}$ and $\tilde{\Omega}_{1}$ such that both have Lipschitz boundary and $\Omega_{1} \subset \tilde{\Omega}_{1} \subset \Omega$ satisfying the following properties. First, $\bar{\gamma} \cup \operatorname{supp}(f)$ is contained in the interior of $\partial \Omega \cap \partial \Omega_{1}$, second, $\operatorname{dist}\left(K_{1} \cup K_{2}, \overline{\tilde{\Omega}}_{1}\right)>0$, and, finally, $\operatorname{dist}\left(\bar{\Omega}_{1}, \partial \tilde{\Omega}_{1} \cap \Omega\right)>0$.

We note that the Cauchy data of $u_{1}$ and $u_{2}$ coincide on $\gamma$, which is an open portion of $\partial \Omega$. Thus, the unique continuation property for harmonic functions implies that $u_{1}=u_{2}$ in $G$ where $G$ is the connected component of $\Omega \backslash\left(K_{1} \cup K_{2}\right)$ such that $\gamma \subset \partial G$. We remark that $G$ contains $\tilde{\Omega}_{1}$ and, consequently, $\partial G$ contains also the support of $f$.

Let us remark that $\int_{\Omega \backslash K_{i}}\left|\nabla u_{i}\right|^{2}=\int_{\operatorname{supp}(f)} f u_{i}$, for any $i=1,2$. We need to prove that

$$
\int_{G}\left|\nabla u_{1}\right|^{2}=\int_{\operatorname{supp}(f)} f u_{1} .
$$

In fact, in such a case, we would have that $\nabla u_{1}=0$ almost everywhere in $\left(\Omega \backslash K_{1}\right) \backslash G$. Since $u_{1}$ is a non-constant harmonic function in $G_{K_{1}}$, its critical set in $G_{K_{1}}$ cannot have positive measure, therefore $\left|G_{K_{1}} \backslash G\right|=0$. Since $u_{1}=u_{2}$ in $G$ and on $\operatorname{supp}(f)$, we have that $\int_{G}\left|\nabla u_{2}\right|^{2}=\int_{\operatorname{supp}(f)} f u_{2}$, as well. Hence, $\nabla u_{2}=0$ almost everywhere in $\left(\Omega \backslash K_{2}\right) \backslash G$ and $\left|G_{K_{2}} \backslash G\right|=0$.

If (4.1) holds, we have that $\left|G_{K_{i}} \backslash G\right|=0$ for any $i=1,2$. Since we know that $u_{1}=u_{2}$ almost everywhere in $G$ and, for any $i=1,2, u_{i}$ is equal to 0 almost everywhere outside $G_{K_{i}}$, it follows immediately that $u_{1}=u_{2}$ almost everywhere in $\Omega$.

Let us fix a cutoff function $\chi$ such that $\chi \in C_{0}^{\infty}\left(\mathbb{R}^{N}\right), 0 \leq \chi \leq 1, \chi \equiv 1$ on a neighbourhood of $\bar{\Omega}_{1}$ and $\chi \equiv 0$ on $\Omega \backslash \tilde{\Omega}_{1}$. Then, we have that

$$
\begin{aligned}
\int_{G}\left|\nabla u_{1}\right|^{2} & =\int_{G} \nabla u_{1} \cdot \nabla\left(\chi u_{1}\right)+\int_{G} \nabla u_{1} \cdot \nabla\left((1-\chi) u_{1}\right) \\
& =\int_{\operatorname{supp}(f)} f u_{1}+\int_{G} \nabla u_{1} \cdot \nabla\left((1-\chi) u_{1}\right) .
\end{aligned}
$$


Thus, in order to prove (4.1), we need to show that $\int_{G} \nabla u_{1} \cdot \nabla\left((1-\chi) u_{1}\right)=0$.

Let $\kappa_{1}$ be the set with zero capacity associated to $K_{1}$ satisfying the properties stated in the definition of the class $\mathcal{A}$. Then, there exists a sequence of $C_{0}^{\infty}\left(\mathbb{R}^{N}\right)$ functions $v_{m}, m \in \mathbb{N}$, satisfying the following properties. First, $0 \leq v_{m} \leq 1, v_{m}$ is identically equal to 1 in $U_{m}$, an open neighbourhood of $\kappa_{1}$, and $v_{m}$ is identically equal to 0 outside $B_{1 / m}\left(\kappa_{1}\right)=\bigcup_{x \in \kappa_{1}} B_{1 / m}(x)$. Second,

$$
\lim _{m \rightarrow \infty} \int_{\mathbb{R}^{N}}\left|\nabla v_{m}\right|^{2}=0
$$

We infer also that $v_{m}$ converges to zero almost everywhere in $\mathbb{R}^{N}$ and also in the $H^{1}\left(\mathbb{R}^{N}\right)$ norm. Then, we have that

$$
\int_{G} \nabla u_{1} \cdot \nabla\left((1-\chi) u_{1}\right)=\int_{G} \nabla u_{1} \cdot \nabla\left((1-\chi) v_{m} u_{1}\right)+\int_{G} \nabla u_{1} \cdot \nabla\left((1-\chi)\left(1-v_{m}\right) u_{1}\right) .
$$

By (4.2), (3.7), and the dominated convergence theorem, we have

$$
\lim _{m \rightarrow \infty} \int_{G} \nabla u_{1} \cdot \nabla\left((1-\chi) v_{m} u_{1}\right)=0
$$

hence our aim is to prove that

$$
\lim _{m \rightarrow \infty} \int_{G} \nabla u_{1} \cdot \nabla\left((1-\chi)\left(1-v_{m}\right) u_{1}\right)=0 .
$$

Let us fix $m \in \mathbb{N}$ and let us consider the following localization procedure. Let $U_{m}^{\prime}$ be an open neighbourhood of $\bar{\Omega}_{1} \cup \kappa_{1}$ such that $(1-\chi)\left(1-v_{m}\right)$ is identically zero on $U_{m}^{\prime}$.

For any $P \in \partial G_{K_{1}} \backslash U_{m}^{\prime}$, we can find a Cartesian coordinate system, positive constants $r$ and $r_{1}$ and a Lipschitz function $\varphi: \mathbb{R}^{N-1} \rightarrow \mathbb{R}$ such that

$$
\partial G_{K_{1}} \cap Q_{r, r_{1}}(P)=\left\{x=\left(x^{\prime}, x_{N}\right) \in Q_{r, r_{1}}(P): x_{N}=\varphi\left(x^{\prime}\right)\right\} .
$$

For such constants $r$ and $r_{1}$, which may depend on $P$, we set $Q(P)=Q_{r, r_{1}}(P)$. Without loss of generality, we can assume that $Q(P)$ lies at a positive distance from $\bar{\Omega}_{1} \cup \kappa_{1}$ and that, for any $x^{\prime} \in Q_{r}^{\prime}\left(P^{\prime}\right)$ we have that $\left|\varphi\left(x^{\prime}\right)-P_{N}\right| \leq r_{1} / 2$. Let us call $Q^{+}(P)=\left\{x=\left(x^{\prime}, x_{N}\right) \in Q(P): x_{N}>\varphi\left(x^{\prime}\right)\right\}$ and $Q^{-}(P)=\left\{x=\left(x^{\prime}, x_{N}\right) \in\right.$ $\left.Q(P): x_{N}<\varphi\left(x^{\prime}\right)\right\}$. Then, two possibilities arise. Either both $Q^{+}(P)$ and $Q^{-}(P)$ are contained in $G_{K_{1}}$ or only one between these two sets is contained in $G_{K_{1}}$. In this second case, we can suppose, up to changing the coordinate system, that such a set is $Q^{-}(P)$.

If $Q^{-}(P) \subset G_{K_{1}}$, then, by a reflection argument, since $\nabla u_{1} \cdot v=0$ on $\{x=$ $\left.\left(x^{\prime}, x_{N}\right) \in Q(P): x_{N}=\varphi\left(x^{\prime}\right)\right\}, u_{1}$ can be extended by continuity onto the whole $Q(P)$, in such a way that such an extension belongs to $H^{1}(Q(P))$. Therefore, by using Fubini's theorem, we can choose, without loss of generality, $r$ and $r_{1}$ 
in such a way that $\left|\nabla u_{1}\right|$ belongs to $L^{2}\left(\partial Q^{-}(P) \backslash \partial G_{K_{1}}\right)$. If also $Q^{+}(P)$ were contained in $G_{K_{1}}$, then we can assume that $\left|\nabla u_{1}\right|$ belongs, at the same time, to $L^{2}\left(\partial Q^{-}(P) \backslash \partial G_{K_{1}}\right)$ and to $L^{2}\left(\partial Q^{+}(P) \backslash \partial G_{K_{1}}\right)$. The positive constants $r, r_{1}$ and the orientation of the cube for which all the previous properties are satisfied clearly depend on $P$. We shall assume in the sequel that the cube $Q(P)$ satisfies all the previously stated assumptions.

By the compactness of $\partial G_{K_{1}} \backslash U_{m}^{\prime}$, we can find a finite number of points $P_{i}$, $i=1, \ldots, n$, such that $\bigcup_{i=1}^{n} Q\left(P_{i}\right)=U_{m}^{\prime \prime}$ covers $\partial G_{K_{1}} \backslash U_{m}^{\prime}$. Let $U_{m}^{\prime \prime \prime}$ be an open neighbourhood of $G_{K_{1}} \backslash\left(U_{m}^{\prime} \cup U_{m}^{\prime \prime}\right)$ compactly contained in $G_{K_{1}}$.

Thus, $V_{0}=U_{m}^{\prime}, V_{i}=Q\left(P_{i}\right), i=1, \ldots, n$, and $V_{n+1}=U_{m}^{\prime \prime \prime}$ are an open covering of $\bar{G}_{K_{1}}$. Let $\chi_{i} \in C_{0}^{\infty}\left(V_{i}\right), i=0, \ldots, n+1$, be a partition of unity over $\bar{G}_{K_{1}}$, that is each $\chi_{i}$ is nonnegative, $\sum_{i=0}^{n+1} \chi_{i}(x) \leq 1$ for any $x \in \mathbb{R}^{N}$ and $\sum_{i=0}^{n+1} \chi_{i}(x)=1$ for any $x \in \bar{G}_{K_{1}}$.

Then,

$$
\int_{G} \nabla u_{1} \cdot \nabla\left((1-\chi)\left(1-v_{m}\right) u_{1}\right)=\sum_{i=0}^{n+1} \int_{G} \nabla u_{1} \cdot \nabla\left((1-\chi)\left(1-v_{m}\right) \chi_{i} u_{1}\right) .
$$

Let us treat each of the integrals on the right-hand side separately.

Since $(1-\chi)\left(1-v_{m}\right) \chi_{0} u_{1}$ is identically equal to zero, then obviously $\int_{G} \nabla u_{1}$. $\nabla\left((1-\chi)\left(1-v_{m}\right) \chi_{0} u_{1}\right)=0$.

For what concerns $i=n+1$, we notice that $(1-\chi)\left(1-v_{m}\right) \chi_{n+1} u_{1}$ belongs to $C_{0}^{\infty}\left(V_{n+1}\right)$, where $V_{n+1}$ is compactly contained in $G_{K_{1}}$. Let us consider the function $w$ so defined. Let $w$ be equal to $(1-\chi)\left(1-v_{m}\right) \chi_{n+1} u_{1}$ inside $G \cap V_{n+1}$ and let $w$ be equal to zero everywhere else. We have that such a function $w$ belongs to $H^{1}\left(\Omega \backslash K_{2}\right)$ and is equal to zero on $\operatorname{supp}(f)$. Therefore

$$
0=\int_{\Omega \backslash K_{2}} \nabla u_{2} \cdot \nabla w=\int_{G} \nabla u_{1} \cdot \nabla\left((1-\chi)\left(1-v_{m}\right) \chi_{n+1} u_{1}\right) .
$$

Hence, we infer that

$$
\int_{G} \nabla u_{1} \cdot \nabla\left((1-\chi)\left(1-v_{m}\right) u_{1}\right)=\sum_{i=1}^{n} \int_{G} \nabla u_{1} \cdot \nabla\left((1-\chi)\left(1-v_{m}\right) \chi_{i} u_{1}\right) .
$$

We can now restrict our attention to $i \in\{1, \ldots, n\}$, since the other terms give no contribution. Let us fix $i \in\{1, \ldots, n\}$ and let us assume that, up to a rigid tranformation, we have $Q=Q_{r, r_{1}}=Q\left(P_{i}\right)$, for some positive $r$ and $r_{1}$. We set $w_{i}=(1-\chi)\left(1-v_{m}\right) \chi_{i} u_{1}$. Then, let $Q^{-}=Q^{-}\left(P_{i}\right)$ be contained in $G_{K_{1}}$ and let us evaluate $\int_{Q^{-} \cap G} \nabla u_{1} \cdot \nabla w_{i}$. If also $Q^{+}=Q^{+}\left(P_{i}\right)$ is contained in $G_{K_{1}}$ we can treat $\int_{Q^{+} \cap G} \nabla u_{1} \cdot \nabla w_{i}$ in a completely analogous way.

Let $\varepsilon_{0}, 0<\varepsilon_{0}<\min \left\{r / 2, r_{1} / 2\right\}$, be such that $\chi_{i}$ is identically equal to zero outside $Q_{r-\varepsilon_{0}, r_{1}-\varepsilon_{0}}$. For any $\varepsilon \geq 0$, let $\gamma_{\varepsilon}=\left\{x=\left(x^{\prime}, x_{N}\right) \in Q_{r-\varepsilon_{0}, r_{1}-\varepsilon_{0}}: x_{N}=\right.$ 
$\left.\varphi\left(x^{\prime}\right)-\varepsilon\right\}$ and $D_{\varepsilon}=\left\{x=\left(x^{\prime}, x_{N}\right) \in Q_{r-\varepsilon_{0}, r_{1}-\varepsilon_{0}}: x_{N}<\varphi\left(x^{\prime}\right)-\varepsilon\right\}$. We have that, for any $\varepsilon>0, D_{\varepsilon}$ is an open set which is compactly contained in $G_{K_{1}}$. Furthermore, we have that

$$
\lim _{\varepsilon \rightarrow 0^{+}}\left|D_{0} \backslash D_{\varepsilon}\right|=0,
$$

hence

$$
\int_{Q^{-} \cap G} \nabla u_{1} \cdot \nabla w_{i}=\lim _{\varepsilon \rightarrow 0^{+}} \int_{D_{\varepsilon} \cap G} \nabla u_{1} \cdot \nabla w_{i} .
$$

The following two lemmas are crucial steps in order to continue and conclude the proof of Theorem 3.3.

Lemma 4.1. Under the previous hypotheses and notation, we have that

$$
\lim _{\varepsilon \rightarrow 0^{+}} \int_{\gamma_{\varepsilon}}\left|\nabla u_{1} \cdot \nu\right| \mathrm{d} \mathcal{H}^{N-1}=0 .
$$

Proof. Let us consider the domain $Q^{-}$. First of all we notice that it is a domain with Lipschitz boundary and such that its complement and its boundary are connected. Let $M_{0}>0$ be the Lipschitz constant of $\varphi$ and let us fix $M_{1}>\sqrt{1+M_{0}^{2}}$. For any $M>1$ and any $P \in \partial Q^{-}$, let

$$
C_{M}(P)=\left\{x \in Q^{-}:|x-P|<M \operatorname{dist}\left(x, \partial Q^{-}\right)\right\} .
$$

For any $P \in \partial Q^{-}$, let the nontangential maximal function $M(v)$ of a function $v$ defined on $Q^{-}$be given by

$$
M(v)(P)=\sup \left\{|v|(x): x \in C_{M_{1}}(P)\right\} .
$$

We say that $v$ has nontangential limits $\mathcal{H}^{N-1}$-almost everywhere on $\partial Q^{-}$if, for $\mathcal{H}^{N-1}$-almost every $P \in \partial Q^{-}$, we have that

$$
\lim _{\substack{x \rightarrow P \\ x \in C_{M}(P)}} v(x)
$$

exists and is finite for all $M>1$.

Let us remark that $u_{1}$ satisfies, in the weak sense, the following boundary-value problem

$$
\Delta u_{1}=0 \text { in } Q^{-}, \quad \nabla u_{1} \cdot v=\tilde{f} \text { on } \partial Q^{-},
$$

where, by the construction of $Q^{-}, \tilde{f} \in L^{2}\left(\partial Q^{-}\right)$and $\tilde{f}=0$ on $\gamma_{0}$.

Then, by Theorem 2 in [15], we infer that $M\left(\nabla u_{1}\right) \in L^{2}\left(\partial Q^{-}\right)$and that $\nabla u_{1}$ converges nontangentially $\mathcal{H}^{N-1}$-almost everywhere on $\partial Q^{-}$to a function $\nabla u_{1} \in$ $L^{2}\left(\partial Q^{-}\right)$such that $\nabla u_{1}(P) \cdot v(P)=\tilde{f}(P)$ for $\mathcal{H}^{N-1}$-almost every $P \in \partial Q^{-}$.

For every $x^{\prime} \in Q_{r-\varepsilon_{0}}^{\prime}$, if we take $P=\left(x^{\prime}, \varphi\left(x^{\prime}\right)\right)$ and $P_{\varepsilon}=\left(x^{\prime}, \varphi\left(x^{\prime}\right)-\varepsilon\right)=$ $P-\varepsilon e_{N}$, where $e_{N}=\left(0^{\prime}, 1\right) \in \mathbb{R}^{N-1} \times \mathbb{R}$, then $P \in \gamma_{0}$ and $P_{\varepsilon} \in \gamma_{\varepsilon}$. There exists $\varepsilon_{1}>0$ such that, for every $\varepsilon, 0<\varepsilon<\varepsilon_{1}, P_{\varepsilon} \in C_{M_{1}}(P)$. 
Obviously, we have that

$$
\int_{\gamma_{\varepsilon}}\left|\nabla u_{1} \cdot \nu\right|^{2}\left(P_{\varepsilon}\right) \mathrm{d} \mathcal{H}^{N-1}\left(P_{\varepsilon}\right)=\int_{\gamma_{0}}\left|\nabla u_{1} \cdot \nu\right|^{2}\left(P-\varepsilon e_{N}\right) \mathrm{d} \mathcal{H}^{N-1}(P) .
$$

Furthermore, $P_{\varepsilon} \in C_{M_{1}}(P)$ implies that $\left|\nabla u_{1}\right|^{2}\left(P_{\varepsilon}\right) \leq M^{2}(\nabla u)(P)$ for $\mathcal{H}^{N-1}$ almost every $P \in \gamma_{0}$. Since $M^{2}(\nabla u) \in L^{1}\left(\gamma_{0}\right)$, and $\left(\nabla u_{1} \cdot v\right)\left(P-\varepsilon e_{N}\right) \rightarrow 0$ as $\varepsilon \rightarrow 0^{+}$for $\mathcal{H}^{N-1}$-almost every $P \in \gamma_{0}$, then by the dominated convergence theorem we infer that

$$
\int_{\gamma_{\varepsilon}}\left|\nabla u_{1} \cdot v\right|^{2} \mathrm{~d} \mathcal{H}^{N-1} \rightarrow 0, \quad \text { as } \varepsilon \rightarrow 0^{+} .
$$

We remark that $\left[\gamma_{\varepsilon}\right]$ is constant, so the proof can be easily concluded using Hölder inequality.

Lemma 4.2. Let $D$ be an open set compactly contained in $G_{K_{1}}$ such that [əD] is finite. Let $\tilde{D}$ be the intersection of $D$ with $G$. Then, for any $v \in C^{\infty}\left(G_{K_{1}}\right)$ we have

$$
\int_{\partial^{*} \tilde{D} \backslash \partial D}\left(\nabla u_{1} \cdot v\right) v \mathrm{~d} \mathcal{H}^{N-1}=0 .
$$

Proof. We begin by observing that, since $\partial \tilde{D}$ is contained in $\partial D \cup\left(\partial G_{K_{2}} \cap D\right)$, we have that $\tilde{D}$ is a set of finite perimeter. We also recall that $u_{1}=u_{2}$ on $G$ and consequently on $\tilde{D}$.

Let $\chi$ be a $C_{0}^{\infty}(D)$ function. We call $\tilde{v}$ the function which is equal to $\chi v$ on $\tilde{D}$ and is 0 elsewhere. We have that $\tilde{v}$ belongs to $H^{1}\left(\Omega \backslash K_{2}\right)$ and it is equal to 0 on $\operatorname{supp}(f)$. Therefore,

$$
0=\int_{\Omega \backslash K_{2}} \nabla u_{2} \cdot \nabla \tilde{v}=\int_{G} \nabla u_{1} \cdot \nabla \tilde{v}=\int_{\tilde{D}} \nabla u_{1} \cdot \nabla(\chi v) .
$$

Since both $u_{1}$ and $\tilde{v}$ belong to $C^{\infty}(\overline{\tilde{D}})$, by Theorem 2.3 we have that

$$
\int_{\tilde{D}} \nabla u_{1} \cdot \nabla(\chi v)=\int_{\partial^{*} \tilde{D}}\left(\nabla u_{1} \cdot v\right) \chi v \mathrm{~d} \mathcal{H}^{N-1}=\int_{\partial^{*} \tilde{D} \backslash \partial D}\left(\nabla u_{1} \cdot v\right) \chi v \mathrm{~d} \mathcal{H}^{N-1} .
$$

We have obtained that $\int_{\partial^{*} \tilde{D} \backslash \partial D}\left(\nabla u_{1} \cdot v\right) \chi v \mathrm{~d} \mathcal{H}^{N-1}=0$ for any $\chi \in C_{0}^{\infty}(D)$. Using a sequence of functions $\chi$ converging to 1 pointwise in $D$, the lemma follows immediately by the dominated convergence theorem.

Let us fix as before $i \in\{1, \ldots, n\}$. Under the previous notation, we recall that, see (4.6),

$$
\int_{Q^{-} \cap G} \nabla u_{1} \cdot \nabla w_{i}=\lim _{\varepsilon \rightarrow 0^{+}} \int_{D_{\varepsilon} \cap G} \nabla u_{1} \cdot \nabla w_{i},
$$


where $w_{i}=(1-\chi)\left(1-v_{m}\right) \chi_{i} u_{1}$. We notice that $D_{\varepsilon}$ is compactly contained in $G \subset G_{K_{1}}$ and, since it is a domain with Lipschitz boundary, we have that $\left[\partial D_{\varepsilon}\right]$ is finite. Then $D_{\varepsilon} \cap G=\tilde{D}_{\varepsilon}$ is a set of finite perimeter which is compactly contained in $G_{K_{1}}$. Obviously, we have that both $u_{1}$ and $w_{i}$ belong to $C^{\infty}\left(G_{K_{1}}\right)$, hence also to $C^{\infty}\left(\tilde{\tilde{D}}_{\varepsilon}\right)$. By the Gauss-Green formula for sets of finite perimeter, Theorem 2.3, we have that

$$
\int_{\tilde{D}_{\varepsilon}} \nabla u_{1} \cdot \nabla w_{i}=\int_{\partial^{*} \tilde{D}_{\varepsilon}}\left(\nabla u_{1} \cdot v\right) w_{i} \mathrm{~d} \mathcal{H}^{N-1} .
$$

Since $\chi_{i}=0$ on $\partial D_{\varepsilon} \backslash \gamma_{\varepsilon}$, and by Lemma 4.2, we obtain that

$$
\int_{\partial^{*} \tilde{D}_{\varepsilon}}\left(\nabla u_{1} \cdot v\right) w_{i} \mathrm{~d} \mathcal{H}^{N-1}=\int_{\left(\partial^{*} \tilde{D}_{\varepsilon} \cap \gamma_{\varepsilon}\right)}\left(\nabla u_{1} \cdot v\right) w_{i} \mathrm{~d} \mathcal{H}^{N-1} .
$$

Hence,

$$
\left|\int_{\tilde{D}_{\varepsilon}} \nabla u_{1} \cdot \nabla w_{i}\right| \leq \int_{\left(\partial^{*} \tilde{D}_{\varepsilon} \cap \gamma_{\varepsilon}\right)}\left|\nabla u_{1} \cdot v \| u_{1}\right| \mathrm{d} \mathcal{H}^{N-1} .
$$

Obviously $\left(\partial^{*} \tilde{D}_{\varepsilon} \cap \gamma \varepsilon\right)$ is contained in $\gamma_{\varepsilon}$. By Proposition 3.1, in particular by (3.7), we have that $\left|u_{1}\right|$ is uniformly bounded by $C_{1}\|f\|_{L^{p}(\partial \Omega)}$. It follows that

$$
\left|\int_{\tilde{D}_{\varepsilon}} \nabla u_{1} \cdot \nabla w_{i}\right| \leq C_{1}\|f\|_{L^{p}(\partial \Omega)} \int_{\gamma_{\varepsilon}}\left|\nabla u_{1} \cdot v\right| \mathrm{d} \mathcal{H}^{N-1} .
$$

Therefore, by (4.7) and (4.6), we can conclude that $\int_{G} \nabla u_{1} \cdot \nabla\left((1-\chi)\left(1-v_{m}\right) \chi_{i} u_{1}\right)=$ 0 for any $i \in\{1, \ldots, n\}$. Then, by (4.4), we have that $\int_{G} \nabla u_{1} \cdot \nabla((1-\chi)(1-$ $\left.\left.v_{m}\right) u_{1}\right)=0$ for any $m \in \mathbb{N}$, thus (4.3) holds true and the proof is concluded.

\section{Proof of Theorem 3.4}

In this section we briefly sketch the modifications to the proof of Theorem 3.3 which are needed to prove Theorem 3.4.

We begin with the following remark. Let $K$ be an admissible defect belonging to $\mathcal{B}$, with $\partial G_{K}=\bigcup_{i=0}^{n} \gamma_{i}$, where $\gamma_{i}, i=0, \ldots, n$, are pairwise disjoint closed continua. Without loss of generality, we may assume that $\gamma_{0}$ is the continuum containing $\tilde{\gamma}_{0}$. Let $u \in H^{1}\left(G_{K}\right)$ be the solution to (3.2). We remark that we have, in the weak sense, $\int_{\partial \gamma_{i}} \nabla u_{1} \cdot v=0$ for any $i=0,1, \ldots, n$. Here, whenever a part of $\gamma_{i}$ can be reached from both sides by points belonging to $G_{K}, \partial \gamma_{i}$ has to be intended as on either sides of $\gamma_{i}$.

Therefore, by using arguments developed in [2], we can show that there exists a single-valued harmonic function $v$ conjugate to $u$ on the whole $G_{K}$, that is there exists $v \in H^{1}\left(G_{K}\right)$ such that

$$
\nabla v=\left[\begin{array}{rr}
0 & -1 \\
1 & 0
\end{array}\right] \nabla u \quad \text { in } G_{K} .
$$


Such a harmonic function $v$, which is defined up to an additive constant, satisfies a Dirichlet type boundary condition on $\partial G_{K}$, namely we have the following properties. For any $i \neq 0,\left.v\right|_{\gamma_{i}}=c_{i}$, whereas $\left.v\right|_{\gamma_{0} \backslash \tilde{\gamma}_{0}}=c_{0}$, where $c_{i}, i=0, \ldots, n$, are not prescribed constants. On $\tilde{\gamma}_{0}$, we have $v=F$ where $F=\int f(s) \mathrm{d} s$ is an antiderivative of $f$ along $\tilde{\gamma}_{0}$. Here the indefinite integral is taken with respect to arclength on $\tilde{\gamma}_{0}$ in the counterclockwise direction whenever $G_{K}$ lies on the left of $\tilde{\gamma}_{0}$, in the clockwise direction otherwise. Furthermore, a no flux condition of the following kind is satisfied. For every smooth closed and simple curve $\gamma$ contained in $G_{K}$, we have $\int_{\gamma} \nabla v \cdot v=0$. We also recall that such a function $v$ is continuous up to $\bar{G}_{K}$.

Let us begin with the case in which $K_{2}$ belongs to $\mathcal{B}$. Let $v_{1}$ and $v_{2}$ be the harmonic conjugates of $u_{1}$ and $u_{2}$, respectively. We can choose the additive constants in such a way to guarantee that $v_{1}=v_{2}$ almost everywhere in $\tilde{\Omega}_{1}$. By unique continuation and continuity, we also have that $v_{1}=v_{2}$ on $\bar{G}$. Let us assume, by contradiction, that $G_{K_{1}} \backslash \bar{G}$ has positive measure. Let $G_{1}$ be a connected component of $G_{K_{1}} \backslash \bar{G}$. We have that $\partial G_{1} \subset\left(\partial G_{K_{1}} \cup \partial G_{K_{2}}\right) \backslash \tilde{\gamma}_{0}$. By the continuity of $v_{1}$ and $v_{2}$, we can conclude that $v_{1}$ is constant on any connected component of $\partial G_{1}$. The no flux condition and the maximim principle allow us to conclude that $v_{1}$ is constant on $G_{1}$ which is impossible. Thus $\left|G_{K_{1}} \backslash \bar{G}\right|=0$. On the other hand, $\partial G \cap G_{K_{1}}$ has also measure zero. Otherwise, we would have a set of positive measure where $v_{1}$ is constant. Hence we conclude that $\left|G_{K_{1}} \backslash G\right|=0$. By reversing the role of $K_{1}$ and $K_{2}$, we can conclude that $\left|G_{K_{1}} \backslash G\right|=\left|G_{K_{2}} \backslash G\right|=0$ and so in the first case the proof is concluded.

Let us now turn our attention to the case in which $K_{2}$ is an admissible defect such that $\left[\partial G_{K_{2}}\right]$ is finite. In this case the following lemma is the main step of the proof.

Lemma 5.1. There exists a sequence of open sets $D_{j}, j \in \mathbb{N}$, such that, for every $j$, $D_{j} \subset D_{j+1} \subset \subset G_{K_{1}}$, [ə $\left.D_{j}\right]$ is finite, and the following two properties are satisfied

$$
\begin{aligned}
& \lim _{j \rightarrow \infty}\left|G_{K_{1}} \backslash\left(D_{j} \cup \Omega_{1}\right)\right|=0, \\
& \lim _{j \rightarrow \infty} \int_{\partial D_{j} \backslash \bar{\Omega}_{1}}\left|\nabla u_{1} \cdot v\right| \mathrm{d} \mathcal{H}^{N-1}=0 .
\end{aligned}
$$

Proof. Let $v_{1}$ be the harmonic conjugate function to $u_{1}$. By the Sard Lemma, we can find a sequence $\left\{\delta_{j}\right\}_{j \in \mathbb{N}}$ such that, for any $j \in \mathbb{N}, 0<\delta_{j+1}<\delta_{j}<1 / j$ and, for any $i=0,1, \ldots, n$, the values $c_{i}-\delta_{j}$ and $c_{i}+\delta_{j}$ are not critical values of $v_{1}$.

Let us fix $j \in \mathbb{N}$. Let $G_{j}=G_{K_{1}} \backslash\left\{x \in G_{K_{1}}: c_{i}-\delta_{j} \leq v_{1}(x) \leq\right.$ $c_{i}+\delta_{j}$, for some $\left.i=0,1, \ldots, n\right\}$ and $D_{j}=G_{j} \backslash \bar{\Omega}_{1}$. Obviously we have that $D_{j} \subset D_{j+1}$. The continuity of $v_{1}$ on $\bar{G}_{K_{1}}$ implies that every $D_{j}$ is indeed compactly contained in $G_{K_{1}}$. Since a harmonic function can be constant only on sets of measure zero, we conclude that $\left|G_{K_{1}} \backslash G_{j}\right|$ goes to 0 as $j$ goes to $\infty$, hence, since $\left|\partial \Omega_{1}\right|=0,(5.1)$ holds. 
It remains to prove that $\left[\partial D_{j}\right]$ is finite and that (5.2) holds. Let us investigate the structure of $\partial D_{j}$. We have that $\partial D_{j}$ is contained in the union of $\partial \Omega_{1}$, which has finite $\mathcal{H}^{N-1}$ measure, and the level sets $\left\{v_{1}=c_{i} \pm \delta_{j}\right\}$. By compactness and by the fact that we have chosen values which are not critical, we obtain that, for any $i$ and $j, \partial D_{j} \cap\left\{v_{1}=c_{i} \pm \delta_{j}\right\}$ is composed by the restriction to $\bar{D}_{j}$ of a finite number of pairwise disjoint simple analytic curves. Hence, we conclude that $\left[\partial D_{j}\right]$ is finite.

Let us prove that (5.2) holds. We have that $\partial D_{j} \backslash \partial \Omega_{1}=\partial D_{j} \backslash \bar{\Omega}_{1}$ is contained in $\left\{v_{1}=c_{i} \pm \delta_{j}\right\} \cap \bar{D}_{j}$. On each analytic curve $\gamma$ contained in $\left\{v_{1}=c_{i} \pm \delta_{j}\right\}$, we have that $v_{1}$ is constant, hence there $\nabla v_{1}$ is parallel to $v$, the normal to $\gamma$. By the definition of harmonic conjugate, we infer that $\nabla u_{1} \cdot v=0$ on each of these curves $\gamma$, therefore we have obtained that

$$
\int_{\partial D_{j} \backslash \bar{\Omega}_{1}}\left|\nabla u_{1} \cdot v\right| \mathrm{d} \mathcal{H}^{N-1}=0, \quad \text { for any } j \in \mathbb{N},
$$

and the proof is concluded.

By using the notation introduced in the previous section, we recall that, in order to conclude the proof, it is enough to show that $\int_{G} \nabla u_{1} \cdot \nabla\left((1-\chi) u_{1}\right)=0$. By Lemma 5.1, in particular by (5.1), we notice that

$$
\int_{G} \nabla u_{1} \cdot \nabla\left((1-\chi) u_{1}\right)=\lim _{j \rightarrow \infty} \int_{D_{j} \cap G} \nabla u_{1} \cdot \nabla\left((1-\chi) u_{1}\right) .
$$

We recall that $D_{j}$ is compactly contained in $G_{K_{1}}$ and $\left[\partial D_{j}\right]$ is finite. Let $\tilde{D}_{j}=$ $D_{j} \cap G$. Obviously, we have that both $u_{1}$ and $(1-\chi) u_{1}$ belong to $C^{\infty}\left(G_{K_{1}}\right)$. By the Gauss-Green formula for sets of finite perimeter, Theorem 2.3, we have that

$$
\int_{\tilde{D}_{j}} \nabla u_{1} \cdot \nabla\left((1-\chi) u_{1}\right)=\int_{\partial^{*} \tilde{D}_{j}}\left(\nabla u_{1} \cdot v\right)\left((1-\chi) u_{1}\right) \mathrm{d} \mathcal{H}^{N-1} .
$$

Since $\chi=1$ on $\partial \Omega_{1}$, and by Lemma 4.2, we obtain that

$$
\int_{\partial^{*} \tilde{D}_{j}}\left(\nabla u_{1} \cdot v\right)\left((1-\chi) u_{1}\right) \mathrm{d} \mathcal{H}^{N-1}=\int_{\left(\partial^{*} \tilde{D}_{j} \cap \partial D_{j}\right) \backslash \partial \Omega_{1}}\left(\nabla u_{1} \cdot v\right)\left((1-\chi) u_{1}\right) \mathrm{d} \mathcal{H}^{N-1} .
$$

Obviously $\left(\partial^{*} \tilde{D}_{j} \cap \partial D_{j}\right) \backslash \partial \Omega_{1}$ is contained in $\partial D_{j} \backslash \partial \Omega_{1}$. We conclude, by (5.2), that $\int_{\left(\partial^{*} \tilde{D}_{j} \cap \partial D_{j}\right) \backslash \partial \Omega_{1}}\left(\nabla u_{1} \cdot v\right)\left((1-\chi) u_{1}\right) \mathrm{d} \mathcal{H}^{N-1}=0$. Therefore, $\int_{G} \nabla u_{1} \cdot \nabla((1-$ $\left.\chi) u_{1}\right)=0$ and the proof is concluded.

\section{Stability results}

In this section we limit ourselves to the three dimensional case, however adaptations of these results to the two-dimensional case can be easily inferred. We remark 
that, in two dimensions, using duality arguments similar to the one used to prove Theorem 3.4, other, and stronger, results of stability may be obtained, we refer to [6] for a discussion on this issue. We also recall that a similar result, for what concerns the stability of the direct problem with respect to the variation of $K$, has been obtained in [12].

Let $T$ be the closed equilateral triangle which is contained in the plane $\pi=$ $\left\{(x, y, z) \in \mathbb{R}^{3}: z=0\right\}$ with vertices $V_{1}=(0,1,0), V_{2}=(-\sqrt{3} / 2,-1 / 2,0)$ and $V_{3}=(\sqrt{3} / 2,-1 / 2,0)$ and $T^{\prime} \subset \mathbb{R}^{2}$ be its projection on the plane $\pi$. Fixed a positive constant $L$, we call an $L$-generalized triangle a set $\Gamma$ such that, up to a rigid transformation, $\Gamma=\left\{(x, y, z) \in \mathbb{R}^{3}:(x, y) \in \varphi\left(T^{\prime}\right), z=\varphi_{1}(x, y)\right\}$, where $\varphi: \mathbb{R}^{2} \rightarrow \mathbb{R}^{2}$ is a bi-Lipschitz function with constant $L$ such that $\varphi(0)=0$ and $\varphi_{1}: \mathbb{R}^{2} \rightarrow \mathbb{R}$ is a Lipschitz map with Lipschitz constant bounded by $L$ and such that $\varphi_{1}(0,0)=0$.

The image through $\varphi$ of any vertex or side of $T^{\prime}$ will be called a vertex or side of $\varphi\left(T^{\prime}\right)$, respectively. The image on the graph of $\varphi_{1}$ of one of the vertices of $\varphi\left(T^{\prime}\right)$ will be called a vertex of $\Gamma$, whereas the image of one of the sides of $\varphi\left(T^{\prime}\right)$ will be called a side of $\Gamma$.

We remark that there exists a constant $L_{1}>0$, depending on $L$ only, such that we can find $\varphi_{2}: \mathbb{R}^{3} \rightarrow \mathbb{R}^{3}$, a bi-Lipschitz function with constant $L_{1}$, such that $\Gamma=\varphi_{2}(T)$.

We introduce the following class of defects. Let $\Omega, \Omega_{1}$ and $\tilde{\Omega}_{1}$ be three bounded domains with Lipschitz boundary such that $\Omega_{1} \subset \tilde{\Omega}_{1} \subset \Omega$ and the following properties are satisfied. First, $\Omega \backslash \tilde{\Omega}_{1}$ is not empty. Then, there exists $\gamma$, an open subset of $\partial \Omega$, such that $\bar{\gamma}$ is contained in the interior of $\partial \Omega_{1} \cap \partial \Omega$. Furthermore, $\operatorname{dist}\left(\bar{\Omega}_{1}, \partial \tilde{\Omega}_{1} \cap \Omega\right)>0$.

Let us fix positive constants $L, \delta, c, 0<c<1$, a strictly increasing function $\omega:(0,+\infty) \rightarrow(0,+\infty)$ such that $\omega(s) \leq s$ for any $s \in(0,+\infty)$, and a point $x_{0} \in \Omega_{1}$. Then, we say that a defect $K \subset \bar{\Omega}$ belongs to the class of admissible defects $\mathcal{C}$ whenever the following conditions are satisfied.

i) $\operatorname{dist}\left(K, \overline{\tilde{\Omega}}_{1}\right) \geq \delta$;

ii) $\partial G_{K}=\bigcup_{i=1}^{n} \Gamma_{i}$, where $n$ depends on $K$ and each $\Gamma_{i}$ is an $L$-generalized triangle;

iii) for any $i, j \in\{1, \ldots, n\}$ with $i \neq j$, we have that either $\Gamma_{i} \cap \Gamma_{j}$ is not empty or $\operatorname{dist}\left(\Gamma_{i}, \Gamma_{j}\right) \geq \delta$

iv) for any $i, j \in\{1, \ldots, n\}$ with $i \neq j$, if $\Gamma_{i} \cap \Gamma_{j}$ is not empty then $\Gamma_{i} \cap \Gamma_{j}$ is either a common side $\gamma$ or a common vertex $V$. Furthermore, in such a case, for any $x \in \Gamma_{i}$ we have $\operatorname{dist}\left(x, \Gamma_{j}\right) \geq c \operatorname{dist}(x, \gamma)$ or $\operatorname{dist}\left(x, \Gamma_{j}\right) \geq c|x-V|$, respectively;

v) for any $t>0$ and for any $x_{1} \in G_{K}$ so that $\bar{B}_{t}\left(x_{1}\right)$ is contained in $G_{K}$, we can find a smooth (for instance $C^{1}$ ) curve $\gamma$ connecting $x_{1}$ to $x_{0}$ so that $\bar{B}_{\omega(t)}(\gamma)$ is contained in $G_{K}$ as well.

We make the following comments on the properties of the sets $K$ belonging to the class $\mathcal{C}$. Assumption i) is self-explanatory. For what concerns assumptions ii)-iv), we may think that the sets $\Gamma_{i}, i=1, \ldots, n$, are a kind of triangulation of $\partial G_{K}$. 
If the $\Gamma_{i}$ would be planar we would have a triangulation of the surface $\partial G_{K}$ in the usual sense. Furthermore, we control how the various parts of the triangulation are close to each other and we also require a kind of uniform connectedness property, assumption $\mathrm{v}$ ).

In order to simplify the notation, let us assume that there exists a point $x \in \partial \Omega$ such that $K=\{x\}$ belongs to $\mathcal{C}$. In particular, this is equivalent to have the following assumptions upon $\Omega$ and $\tilde{\Omega}_{1}$. First, there exists $x \in \partial \Omega$ such that $\operatorname{dist}\left(x, \overline{\tilde{\Omega}}_{1}\right) \geq \delta$. Second, we assume that $\partial \Omega$ satisfies assumptions ii)-v). Without loss of generality, we can assume that any $K \in \mathcal{C}$ contains the point $x$. Therefore, we have that $\mathcal{C}$ is not empty and any element of $\mathcal{C}$ is a nonempty compact set. We notice that there exists an integer $M$ such that for any $K \in \mathcal{C}$, such that $\partial G_{K}=\bigcup_{i=1}^{n} \Gamma_{i}$, we have $n \leq M$. We remark that $\mathcal{C} \subset \mathcal{A}$ and that, for any $K \in \mathcal{C}$, $\left[\partial G_{K}\right]$ is bounded by a constant depending on $\mathcal{C}$ only. Let us consider the following compactness result.

Lemma 6.1. The class $\mathcal{C}$ is compact with respect to the Hausdorff distance.

Proof. Let us consider a sequence $K_{m}, m \in \mathbb{N}$, of defects belonging to $\mathcal{C}$. Up to passing to a subsequence, we can assume, without loss of generality, that $\partial G_{K_{m}}=$ $\bigcup_{i=1}^{n} \Gamma_{i}^{m}$, with the same integer $n$ for any $m \in \mathbb{N}$, and that, as $m \rightarrow \infty, K_{m}$ converges to a compact set $K$ in the Hausdorff distance and, for any $i=1, \ldots, n$, $\Gamma_{i}^{m}$ converges to a compact set $\Gamma_{i}$, again in the Hausdorff distance. It is not difficult to show that $K \subset \bar{\Omega}$ and $\operatorname{dist}\left(K, \bar{\Omega}_{1}\right) \geq \delta$. Furthermore, again up to passing to a subsequence, we can also ensure that $\Gamma_{i}$ is an $L$-generalized triangle for any $i=1, \ldots, n$.

We shall prove later that $\partial G_{K}=\bigcup_{i=1}^{n} \Gamma_{i}$. We now concentrate our attention to properties iii) and iv).

If $\operatorname{dist}\left(\Gamma_{i}, \Gamma_{j}\right)<\delta$, then, for any $m \geq m_{0}$, we have $\operatorname{dist}\left(\Gamma_{i}^{m}, \Gamma_{j}^{m}\right)<\delta$. Therefore $\Gamma_{i}^{m} \cap \Gamma_{j}^{m}$ is not empty and, consequently, we deduce that also $\Gamma_{i} \cap \Gamma_{j}$ is not empty, thus iii) is proved.

Let us assume that, for some $i \neq j$, we have $\Gamma_{i} \cap \Gamma_{j} \neq \emptyset$. Then, for any $m \geq m_{0}$, we have $\Gamma_{i}^{m} \cap \Gamma_{j}^{m} \neq \varnothing$. Without loss of generality, up to restricting to a further subsequence, we can assume that, for any $m \geq m_{0}$ either we have $\Gamma_{i}^{m} \cap \Gamma_{j}^{m}=\left\{V_{m}\right\}$, where $V_{m}$ is a common vertex, or $\Gamma_{i}^{m} \cap \Gamma_{j}^{m}=\gamma_{m}$, where $\gamma_{m}$ is a common side. Since sides and vertices of generalized triangles are preserved by the convergence in the Hausdorff distance, we have that $\Gamma_{i} \cap \Gamma_{j}$ is a common vertex $V$ or a common side $\gamma$, respectively. Let $x \in \Gamma_{i}$. Then, there exist $x_{m} \in \Gamma_{i}^{m}$ such that $x_{m} \rightarrow x$ as $m \rightarrow \infty$. Besides, we have that $\operatorname{dist}\left(x_{m}, \Gamma_{j}^{m}\right) \geq c\left|x_{m}-V_{m}\right|$ or $\operatorname{dist}\left(x_{m}, \Gamma_{j}^{m}\right) \geq c \operatorname{dist}\left(x_{m}, \gamma_{m}\right)$, respectively. Since $V_{m} \rightarrow V$ or $\gamma_{m}$ converges to $\gamma$ in the Hausdorff distance, respectively, and $\operatorname{dist}\left(x_{m}, \Gamma_{j}^{m}\right) \rightarrow \operatorname{dist}\left(x, \Gamma_{j}\right)$, we obtain that iv) immediately follows.

We claim that $\chi_{G_{K_{m}}}$ converges almost everywhere to $\chi_{G_{K}}$, as $m \rightarrow \infty$. Here $\chi$ denotes the characteristic function. 
Let $x \in G_{K}$. Then, for any $m \geq m_{0}, x \in A_{m}$ which is a connected component of $\Omega \backslash K_{m}$. Let $\gamma$ be a smooth curve connecting $x$ to $x_{0}$ inside $G_{K}$. Then there exists $r>0$ such that $B_{r}(\gamma) \subset G_{K}$. Therefore, there exists $m_{1}$ such that for every $y \in \gamma$ and any $m \geq m_{1}$, we have $y \in \Omega \backslash K_{m}$. We deduce that $x \in G_{K_{m}}$ for any $m \geq m_{1}$ and that $\chi_{G_{K_{m}}}(x) \rightarrow \chi_{G_{K}}(x)$, as $m \rightarrow \infty$, for any $x \in G_{K}$.

Let $x \in \Omega \backslash\left(\bigcup_{i=1}^{n} \Gamma_{i} \cup G_{K}\right)$. Then there exists $r>0$ such that $B_{r}(x) \subset$ $\Omega \backslash \bigcup_{i=1}^{n} \Gamma_{i}$. Therefore, for any $m \geq m_{0}, \bar{B}_{r / 2}(x) \in A_{m}$ which is a connected component of $\Omega \backslash \partial G_{K_{m}}$. Let us assume, by contradiction, that up to a subsequence we have $A_{m}=G_{K_{m}}$ for every $m$. Then there exists $\gamma_{m}$ connecting $x$ to $x_{0}$ inside $G_{K_{m}}$ such that $\bar{B}_{\omega(r / 2)}\left(\gamma_{m}\right) \subset G_{K_{m}}$. Therefore, for any $m \geq m_{1}, \gamma_{m}$ is contained in $\Omega \backslash K$, thus $x \in G_{K}$ which is a contradiction. Hence, we deduce that for any $m \geq m_{2}, x \notin G_{K_{m}}$ and that $\chi_{G_{K_{m}}}(x) \rightarrow \chi_{G_{K}}(x)$, as $m \rightarrow \infty$, for any $x \in$ $\Omega \backslash\left(\bigcup_{i=1}^{n} \Gamma_{i} \cup G_{K}\right)$.

For any $x \notin \Omega$ we have $\chi_{G_{K_{m}}}(x)=\chi_{G_{K}}(x)=0$ for any $m$. Since $\bigcup_{i=1}^{n} \Gamma_{i}$ has N-dimensional Lebesgue measure zero, the claim is proved. We remark that completely analogous reasonings lead us to the fact that $\partial G_{K}=\bigcup_{i=1}^{n} \Gamma_{i}$.

Let us now prove v). Let $x \in G_{K}$ be such that $\bar{B}_{t}(x) \subset G_{K}$, for some $t>0$. Then, for some $\varepsilon>0$ and $m_{0}$, we have $\bar{B}_{t+\varepsilon}(x) \subset G_{K_{m}}$ for any $m \geq m_{0}$. For any $m \geq m_{0}$, let $\gamma_{m}$ be a smooth curve connecting $x$ to $x_{0}$ such that $\bar{B}_{\omega(t+\varepsilon)}\left(\gamma_{m}\right) \subset$ $G_{K_{m}}$. For any $\varepsilon_{1}, 0<\varepsilon_{1}<\omega(t+\varepsilon)$, there exists $m_{1}$ such that $\bar{B}_{\omega(t+\varepsilon)-\varepsilon_{1}}\left(\gamma_{m}\right) \subset$ $G_{K}$ for any $m \geq m_{1}$. Since $\omega$ is strictly increasing, we can choose $\varepsilon_{1}$ such that $\omega(t+\varepsilon)-\varepsilon_{1} \geq \omega(t)$ an so property $\left.\mathrm{v}\right)$ is proved.

We now formulate our stability result. Let $\gamma$ be an open nonempty subset of $\partial \Omega$ such that $\bar{\gamma}$ is contained in the interior of $\partial \Omega \cap \partial \Omega_{1}$ and let $\tilde{\gamma}$ be a closed subset of the interior of $\partial \Omega \cap \partial \Omega_{1}$. In order to simplify some technicalities of the proofs, we shall make, without loss of generality, the following assumption. We assume that for any $K \in \mathcal{C}$ and any $L$-generalized triangle $\Gamma \subset \partial G_{K}$ such that $\Gamma \cap \partial \Omega \cap \partial \Omega_{1} \neq \varnothing$, we have that either $\Gamma$ is contained in the interior of $\partial \Omega \cap \partial \Omega_{1}$ or $\Gamma \cap(\bar{\gamma} \cup \tilde{\gamma})$ is empty.

For any $n \in \mathbb{N}$, let $f_{n} \in L^{p}(\partial \Omega)$ be such that $\operatorname{supp}\left(f_{n}\right) \subset \tilde{\gamma}$ and $\int_{\partial \Omega} f_{n}=0$. Let $K_{n}$ belong to $\mathcal{C}$ and let $u_{n}=u\left(f_{n}, K_{n}\right)$, where $u(f, K)$ is the solution to (3.2)(3.3)-(3.4). We recall that $u_{n} \in S B V(\Omega)$ and $\nabla u_{n}$ denotes its approximate gradient. We set $g_{n}=\left.u_{n}\right|_{\gamma}$. Then the following stability results hold.

In the first one we treat the stability of the direct problem (3.2)-(3.3)-(3.4) with respect to $K$ (and $f$ ). In the second we deal with the stability of the unique continuation problem with respect to the Cauchy data.

Theorem 6.2. Let us assume that, as $n \rightarrow \infty, f_{n} \rightarrow f$ weakly in $L^{p}(\partial \Omega)$.

If $K_{n}$ converges to $K$ in the Hausdorff distance, then $u_{n}$ converges to $u=$ $u(f, K)$ weakly* in $B V(\Omega)$ and $\nabla u_{n}$ converges to $\nabla u$ in $L^{2}\left(\Omega, \mathbb{R}^{3}\right)$. Furthermore, $\left.g_{n} \rightarrow u\right|_{\gamma}=g$ in $L^{2}(\gamma)$. 
Theorem 6.3. Let us assume that, as $n \rightarrow \infty, f_{n} \rightarrow f$ weakly in $L^{p}(\partial \Omega)$.

If $g_{n} \rightarrow g$ in $L^{2}(\gamma)$, then there exists $K \in \mathcal{C}$ such that $u_{n}$ converges to $u=$ $u(f, K)$ weakly* in $B V(\Omega)$ and $\nabla u_{n}$ converges to $\nabla u$ in $L^{2}\left(\Omega, \mathbb{R}^{3}\right)$. Furthermore, $\left.u\right|_{\gamma}=g$.

We begin with some remarks based on the only assumption that, as $n \rightarrow \infty$, $f_{n} \rightarrow f$ weakly in $L^{p}(\partial \Omega)$. First of all, $u_{n} \in L^{\infty}(\Omega), \nabla u_{n} \in L^{2}\left(\Omega, \mathbb{R}^{3}\right)$ and $\left[S\left(u_{n}\right) \backslash \partial G_{K_{n}}\right]=0$. Furthermore, $\left\|u_{n}\right\|_{L^{\infty}(\Omega)},\left\|\nabla u_{n}\right\|_{L^{2}\left(\Omega, \mathbb{R}^{3}\right)}$ and $\left[\partial G_{K_{n}}\right]$ are uniformly bounded.

The crucial step is contained in the following proposition.

Proposition 6.4. Let us assume that, as $n \rightarrow \infty, f_{n} \rightarrow f$ weakly in $L^{p}(\partial \Omega)$. Let us assume that there exists $u \in B V(\Omega)$ such that $u_{n} \stackrel{*}{\rightarrow} u$ weakly* in $B V(\Omega), u_{n} \rightarrow$ $u$ almost everywhere in $\Omega$ and $\nabla u_{n} \rightarrow \nabla u$ weakly in $L^{2}\left(\Omega, \mathbb{R}^{3}\right)$. Furthermore, we assume that $g_{n} \rightarrow g=\left.u\right|_{\gamma}$ strongly in $L^{2}(\gamma)$, and that there exists $K \in \mathcal{C}$ such that $K_{n}$ converges to $K$ in the Hausdorff distance.

Under these assumptions, $u=u(f, K)$ and $\nabla u_{n} \rightarrow \nabla u$ in $L^{2}\left(\Omega, \mathbb{R}^{3}\right)$.

Let us show that Proposition 6.4 allows us to conclude the proof of Theorems 6.2 and 6.3.

Let us consider Theorem 6.2. From every subsequence we can extract a further subsequence satisfying the assumptions of Proposition 6.4. In fact, with the only assumption that, as $n \rightarrow \infty, f_{n} \rightarrow f$ weakly in $L^{p}(\partial \Omega)$, we can ensure that, up to a subsequence, by Theorem $2.1, u_{n} \stackrel{*}{\rightarrow} u$ weakly* in $B V(\Omega)$, where $u \in S B V(\Omega)$. We can also assume, without loss of generality, that $u_{n} \rightarrow u$ almost everywhere in $\Omega$ and that $\nabla u_{n} \rightarrow \nabla u$ weakly in $L^{2}\left(\Omega, \mathbb{R}^{3}\right)$. Consequently, $u_{n} \rightarrow u$ weakly in $H^{1}\left(\tilde{\Omega}_{1}\right)$ where $u$ is a harmonic function in $\tilde{\Omega}_{1}$. We can further deduce that, by the compactness of the trace operator from $H^{1}\left(\tilde{\Omega}_{1}\right)$ to $L^{2}(\gamma), g_{n} \rightarrow g=\left.u\right|_{\gamma}$ strongly in $L^{2}(\gamma)$, and that on the interior of $\partial \Omega_{1} \cap \partial \Omega$ we have, in the weak sense, $\partial u / \partial v=f$. Finally, by Lemma 6.1 , there exists $K \in \mathcal{C}$ such that $K_{n}$ converges to $K$ in the Hausdorff distance. We apply Proposition 6.4, noting that $K$ might depend on the first subsequence we have extracted. However, $u=u(f, K)$ must satisfy $\left.u\right|_{\gamma}=g$. Hence, by the uniqueness result, Theorem 3.3, $u$ does not depend on the subsequence, hence we can conclude that the whole sequence $u_{n}$ converges in the required way.

The reasoning is similar for Theorem 6.3. From every subsequence we can extract a further subsequence satisfying the assumptions of Proposition 6.4. We apply Proposition 6.4 and we note that $K$ must be the one of the hypothesis, therefore every such a second subsequence converges, in the required sense, to the same $u=u(f, K)$. Thus, the whole sequence $u_{n}$ converges as required.

It remains to prove Proposition 6.4. Before doing it, we need to introduce the following notation.

Let $\sigma$ be the conductivity of the medium occupying the region $D \subset \mathbb{R}^{N}, N \geq$ 2. We make the assumption that $\sigma$ is an $N \times N$ matrix whose entries are measurable 
functions on $D$ satisfying

$$
\begin{aligned}
& 0<\lambda|\xi|^{2} \leq \sigma(x) \xi \cdot \xi \quad \text { for every } \xi \in \mathbb{R}^{N} \text { and for a.e. } x \in \Omega, \\
& \left|\sigma_{i, j}(x)\right| \leq \lambda^{-1} \quad \text { for every } i, j=1, \ldots, N, \text { and for a.e. } x \in \Omega
\end{aligned}
$$

where $\lambda$ is a positive constant less than 1 .

We look for conditions upon which weak solutions to elliptic equations in divergence form in a domain $D$ belong to $H_{\mathrm{loc}}^{1, q}(D)$ with $q>2$.

The following result by N. G. Meyers, [17], states that, for any $\sigma \in L^{\infty}\left(D, M^{N \times N}\right)$ satisfying (6.1) with a constant $\lambda$, this holds for some $q>2$ depending on $\lambda$ and $N$ only.

Theorem 6.5 (Meyers). Let $D$ be a bounded domain with Lipschitz boundary contained in $\mathbb{R}^{N}, N \geq 2$. Fixed $\lambda, 0<\lambda<1$, there exists a constant $Q, 2<Q<\infty$, depending on $\lambda$ and on $N$ only, $Q \rightarrow 2$ as $\lambda \rightarrow 0$ and $Q \rightarrow \infty$ as $\lambda \rightarrow 1$, such that any $\sigma \in L^{\infty}\left(D, M^{N \times N}\right)$ satisfying (6.1) with constant $\lambda$, satisfies the following property.

For any $q, 2<q<Q$, if $h \in L^{q}\left(D, \mathbb{R}^{N}\right), h_{1} \in L^{q}(D)$ and $u \in H^{1}(D)$ is a weak solution to

$$
\operatorname{div}(\sigma \nabla u)=\operatorname{div}(h)+h_{1} \quad \text { in } D
$$

then $u \in H_{\mathrm{loc}}^{1, q}(D)$ and for any $D_{1} \subset \subset D$ the following estimate holds

$$
\|u\|_{H^{1, q}\left(D_{1}\right)} \leq C\left(\|u\|_{H^{1}(D)}+\|h\|_{L^{q}\left(D, \mathbb{R}^{N}\right)}+\left\|h_{1}\right\|_{L^{q}(D)}\right)
$$

where the constant $C$ depends on $\lambda, N, q, D_{1}$ and $D$ only.

Proof of Proposition 6.4. Let us investigate some properties of $u$. First of all, we have that $u$ is harmonic in $G_{K}$ and it is almost everywhere equal to zero outside $G_{K}$. By Proposition 3.1, we also deduce that $u \in L^{\infty}(\Omega)$ and $u_{n} \rightarrow u$ in $L^{2}(\Omega)$. Therefore, by Caccioppoli's inequality, we obtain that $u_{n}$ converges strongly to $u$ in $H^{1}$ on any compact set of $G_{K}$.

Let us show that $u_{n}$ converges strongly to $u$ in $H^{1}\left(\Omega_{1}\right)$. In fact, let $\tilde{u}_{n}$ be the solution to (3.10)-(3.11) with $f$ replaced by $f_{n}$ and let $\tilde{u}$ be the solution to (3.10)(3.11). We have that $\tilde{u}_{n}$ converges to $\tilde{u}$ weakly in $H^{1}\left(\tilde{\Omega}_{1}\right)$. Furthermore,

$$
\int_{\tilde{\Omega}_{1}}\left|\nabla \tilde{u}_{n}\right|^{2}=\int_{\partial \Omega_{1} \cap \partial \Omega} f_{n} \tilde{u}_{n} \rightarrow \int_{\partial \Omega_{1} \cap \partial \Omega} f \tilde{u}=\int_{\tilde{\Omega}_{1}}|\nabla \tilde{u}|^{2} .
$$

Therefore, $\tilde{u}_{n}$ actually converges to $\tilde{u}$ strongly in $H^{1}\left(\tilde{\Omega}_{1}\right)$. 
Let $\hat{u}_{n}=\tilde{u}_{n}-u_{n} \in H^{1}\left(\tilde{\Omega}_{1}\right)$ and $\hat{u}=\tilde{u}-u \in H^{1}\left(\tilde{\Omega}_{1}\right)$. We denote $Q=$ $(-1,1) \times(-1,1) \times(0,1)$ and $S=(-1,1) \times(-1,1) \times\{0\}$ and $Q_{1}=(-1 / 2,1 / 2) \times$ $(-1 / 2,1 / 2) \times(0,1 / 2)$ and $S_{1}=(-1 / 2,1 / 2) \times(-1 / 2,1 / 2) \times\{0\}$. For every $x \in \partial \Omega_{1} \cap \partial \Omega$, by the Lipschitz property of $\partial \Omega$, there exists $r>0$ such that the following properties are satisfied. First, $B_{r}(x) \cap \Omega \subset \tilde{\Omega}_{1}$ and $\partial \Omega \cap B_{r}(x)$ is the graph of a Lipschitz function. Furthermore, we can find a constant $r_{1}, 0<r_{1}<r$, and a bi-Lipschitz function $\varphi: \mathbb{R}^{3} \rightarrow \mathbb{R}^{3}$ such that $B_{r_{1}}(x) \cap \Omega \subset \varphi\left(Q_{1}\right) \subset \varphi(Q) \subset$ $B_{r}(x) \cap \Omega$ and $B_{r_{1}}(x) \cap \partial \Omega \subset \varphi\left(S_{1}\right) \subset \varphi(S) \subset B_{r}(x) \cap \partial \Omega$. Let $v_{n}=\hat{u}_{n} \circ \varphi$. Then, there exists $\sigma \in L^{\infty}\left(Q, M^{3 \times 3}\right)$ satisfying (6.1) for some $\lambda>0$ such that $\operatorname{div}\left(\sigma \nabla v_{n}\right)=0$ in $Q$ and $\sigma \nabla v_{n} \cdot v=0$ on $S$. By a reflection argument and by Theorem 6.5, we infer that there exists $q>2$ such that $v_{n} \in H^{1, q}\left(Q_{1}\right)$ and, consequently, $\left\|\hat{u}_{n}\right\|_{H^{1, q}\left(B_{r_{1}}(x) \cap \Omega\right)} \leq C$, where $r_{1}, q$ and $C$ might depend on $x$ but not on $n$.

By compactness, there exist $x_{1}, \ldots, x_{m} \in \partial \Omega_{1} \cap \partial \Omega$ such that $\partial \Omega_{1} \cap \partial \Omega \subset U=$ $\bigcup_{i=1}^{m} B_{r_{1}\left(x_{i}\right)}\left(x_{i}\right)$. Therefore, there exist $q>2$ and $C$ such that $\left\|\hat{u}_{n}\right\|_{H^{1, q}(U \cap \Omega)} \leq C$ for any $n$. Hence, for any $r>0$ such that $B_{r}\left(\partial \Omega_{1} \cap \partial \Omega\right) \subset U$, we have

$$
\left\|\nabla \hat{u}_{n}\right\|_{L^{2}\left(B_{r}\left(\partial \Omega_{1} \cap \partial \Omega\right) \cap \Omega\right)} \leq\left|B_{r}\left(\partial \Omega_{1} \cap \partial \Omega\right) \cap \Omega\right|^{(q-2) /(2 q)}\left\|\nabla \hat{u}_{n}\right\|_{L^{q}(U \cap \Omega)} .
$$

Since $\hat{u}_{n}$ converges to $\hat{u}$ strongly in $H^{1}$ on any compact subset of $\tilde{\Omega}_{1}$ and $\left\|\nabla \hat{u}_{n}\right\|_{L^{q}(U \cap \Omega)}$ is uniformly bounded, we can easily conclude that $\hat{u}_{n}$ converges to $\hat{u}$ strongly in $H^{1}\left(\Omega_{1}\right)$ and therefore $u_{n}$ converges to $u$ strongly in $H^{1}\left(\Omega_{1}\right)$.

Let us show that $\nabla u_{n}$ converges to $\nabla u$ strongly in $L^{2}(\Omega)$. We know that $\nabla u_{n}$ converges to $\nabla u$ strongly in $L^{2}$ on any compact subset of $G_{K}$. In order to control what happens near the boundary of $G_{K}$, we make use of the following reasoning. Let us consider the set $A$ which is the union of the sides of all generalized triangles belonging to $\partial G_{K}$ and $\partial G_{K_{n}}$, for any $n$. Such a set has zero capacity. Therefore, for any $R>0$ and any $\varepsilon>0$, we can find $r>0$ and a function $\chi_{R, \varepsilon}$ belonging to $C_{0}^{\infty}\left(B_{R}(A)\right)$ such that $\chi_{R, \varepsilon}$ is identically equal to 1 on $B_{r}(A)$ and $\int_{B_{R}(A)}\left|\nabla \chi_{R, \varepsilon}\right|^{2} \leq \varepsilon^{2}$.

Let us fix a cutoff function $\tilde{\chi} \in C_{0}^{\infty}\left(\mathbb{R}^{3}\right)$ such that $0 \leq \tilde{\chi} \leq 1$, $\tilde{\chi}$ is identically equal to 1 in a neighbourhood of $\overline{\Omega \backslash \bar{\Omega}_{1}}$ and $\tilde{\chi}$ is identically equal to 0 in a neighbourhood of $\bar{\gamma} \cup \tilde{\gamma}$. We have that

$$
\begin{aligned}
& \int_{\left(B_{r}(A) \cap \Omega\right) \backslash \bar{\Omega}_{1}}\left|\nabla u_{n}\right|^{2} \leq \int_{\Omega} \tilde{\chi} \chi_{R, \varepsilon}\left|\nabla u_{n}\right|^{2} \\
& \quad=\int_{\Omega} \nabla u_{n} \cdot \nabla\left(\tilde{\chi} \chi_{R, \varepsilon} u_{n}\right)+\int_{\Omega} \tilde{\chi} u_{n} \nabla u_{n} \cdot \nabla \chi_{R, \varepsilon}+\int_{\Omega} \chi_{R, \varepsilon} u_{n} \nabla u_{n} \cdot \nabla \tilde{\chi} .
\end{aligned}
$$

The first term on the right-hand side is equal to zero, hence we deduce that, by the Poincaré inequality,

$$
\int_{\left(B_{r}(A) \cap \Omega\right) \backslash \bar{\Omega}_{1}}\left|\nabla u_{n}\right|^{2} \leq C \varepsilon,
$$

with a constant $C$ independent on $n$. 
Let now $\Gamma$ be one of the generalized triangles of $\partial G_{K_{n}}$, for some $n$. We assume that $\Gamma \cap(\bar{\gamma} \cup \tilde{\gamma})$ is empty. We recall that $\Gamma=\varphi_{2}(T)$ where $\varphi_{2}$ is a bi-Lipschitz function with constant $L_{1}$. For any $s>0$, let $P_{s}^{+}$be the open pyramid with base the triangle $T$ and vertex $(0,0, s)$ and $P_{s}^{-}$be the open pyramid with base the triangle $T$ and vertex $(0,0,-s)$. For any $t, 0<t<1$, we call $P_{s, t}^{+}$the pyramid which is obtained from $P_{s}^{+}$by a dilation, centred in the origin, of ratio $t$.

We can find $s_{0}, 0<s_{0} \leq 1 / 4$ depending on $\mathcal{C}$ only, such that both $\varphi_{2}\left(P_{s_{0}}^{+}\right)$ and $\varphi_{2}\left(P_{s_{0}}^{-}\right)$do not intersect $\partial G_{K_{n}}$. It may happen that only one of or both of these two sets are contained in $G_{K_{n}}$. Let us assume that $\varphi_{2}\left(P_{s_{0}}^{+}\right)$is contained in $G_{K_{n}}$. Let $v_{n}=u_{n} \circ \varphi_{2}$. Then, there exists $\sigma \in L^{\infty}\left(P_{s_{0}}^{+}, M^{3 \times 3}\right)$ satisfying (6.1) for some $\lambda>0$ such that $\operatorname{div}\left(\sigma \nabla v_{n}\right)=0$ in $P_{s_{0}}^{+}$and $\sigma \nabla v_{n} \cdot v=0$ on $T$. By a reflection argument and by Theorem 6.5, we infer that for any $t, 0<t<1$, there exists $q>2$ such that $v_{n} \in H^{1, q}\left(P_{s_{0}, t}^{+}\right)$and, consequently, $\left\|u_{n}\right\|_{H^{1, q}\left(\varphi_{2}\left(P_{s_{0}, t}^{+}\right)\right)} \leq C$, where $q$ and $C$ depend on $\mathcal{C}$ and on $t$ but not on $n$ or on $\Gamma$. If $\varphi_{2}\left(P_{S_{0}}^{-}\right)$is also contained in $G_{K_{n}}$, then we infer in an analogous way that $\left\|u_{n}\right\|_{H^{1, q}\left(\varphi_{2}\left(P_{s_{0}, t}^{-}\right)\right)} \leq C$ as well.

For any generalized triangle $\Gamma \subset \partial G_{K}$ and any $r>0$, let $\Gamma_{r}$ be the set obtained by removing from $\Gamma$ the $r$-ball of its sides. Let us fix $\varepsilon>0$ and $R>0$ and let $r>0$ and $\chi_{R, \varepsilon}$ be defined as before. We observe that, for any $r_{1}, 0<r_{1}<r / 2, G_{K}$ is contained in the union of $\bar{\Omega}_{1}, B_{r}(A), B_{r_{1}}\left(\Gamma_{r}\right)$, for any generalized triangle $\Gamma \subset \partial G_{K}$ such that $\Gamma \cap(\bar{\gamma} \cup \tilde{\gamma})$ is empty, and $D_{r_{1}}$, where $D_{r_{1}}$ is a compact subset of $G_{K}$.

Let us consider one of these generalized triangles $\Gamma \subset \partial G_{K}$ such that $\Gamma \cap(\bar{\gamma} \cup$ $\tilde{\gamma})$ is empty. Let $\Gamma^{n} \subset \partial G_{K_{n}}$ be the generalized triangle such that, as $n \rightarrow \infty$, $\Gamma^{n}$ converges to $\Gamma$ in the Hausdorff distance and let $\varphi_{2}^{n}$ be its corresponding biLipschitz function. Then, there exist $n_{0} \in \mathbb{N}$ and $t, 0<t<1$, such that for any $n \geq n_{0}$ and any $r_{1}, 0<r_{1}<r / 2$, we have $B_{r_{1}}\left(\Gamma_{r}\right) \subset \varphi_{2}^{n}\left(\overline{P_{s_{0}, t}^{+} \cup P_{s_{0}, t}^{-}}\right)$. Therefore, we can find $q>2$ and $C$ such that for any $n \geq n_{0}$ we have

$$
\left\|\nabla u_{n}\right\|_{L^{q}\left(B_{r_{1}}\left(\Gamma_{r}\right) \cap \Omega\right)} \leq C .
$$

Therefore,

$$
\left\|\nabla u_{n}\right\|_{L^{2}\left(B_{r_{1}}\left(\Gamma_{r}\right) \cap \Omega\right)} \leq C\left|B_{r_{1}}(\Gamma)\right|^{(q-2) /(2 q)},
$$

where $C$ and $q$ do not depend on $n$ and $r_{1}$.

Therefore, for any $\varepsilon>0$ and $R>0$ we can find $r>0, r_{1}>0, C>0$ and $n_{0} \in \mathbb{N}$ such that

$$
\left\|\nabla u_{n}\right\|_{L^{2}\left(\Omega \backslash\left(\bar{\Omega}_{1} \cup D_{r_{1}}\right)\right)} \leq C \varepsilon
$$

for any $n \geq n_{0}$. Since $\nabla u_{n}$ converges to $\nabla u$ strongly in $L^{2}\left(D_{r_{1}} \cup \Omega_{1}\right)$, we immediately infer that $\nabla u_{n}$ converges to $\nabla u$ strongly in $L^{2}(\Omega)$.

What remains to be proved is the fact that $u=u(f, K)$, that is that we have

$$
\int_{G_{K}} \nabla u \cdot \nabla v=\int_{\tilde{\gamma}} f v \quad \text { for any } v \in H^{1}\left(G_{K}\right) .
$$

Let us fix $v \in H^{1}\left(G_{K}\right) \cap L^{\infty}\left(G_{K}\right)$. Let $\tilde{\chi}$ be the cutoff function defined before. 
Then

$\int_{G_{K}} \nabla u \cdot \nabla v=\int_{G_{K}} \nabla u \cdot \nabla(\tilde{\chi} v)+\int_{G_{K}} \nabla u \cdot \nabla((1-\tilde{\chi}) v)=\int_{G_{K}} \nabla u \cdot \nabla(\tilde{\chi} v)+\int_{\tilde{\gamma}} f v$.

We need to show that $\int_{G_{K}} \nabla u \cdot \nabla(\tilde{\chi} v)=0$. First, for any $R>0$ and any $\varepsilon>0$, let $\chi_{R, \varepsilon}$ be defined as before. Then, denoting $\tilde{v}=\tilde{\chi} v$, we have

$$
\int_{G_{K}} \nabla u \cdot \nabla \tilde{v}=\int_{G_{K}} \nabla u \cdot \nabla\left(\chi_{R, \varepsilon} \tilde{v}\right)+\int_{G_{K}} \nabla u \cdot \nabla\left(\left(1-\chi_{R, \varepsilon}\right) \tilde{v}\right) .
$$

We notice that

$$
\left|\int_{G_{K}} \nabla u \cdot \nabla\left(\chi_{R, \varepsilon} \tilde{v}\right)\right| \leq\|\nabla u\|_{L^{2}\left(G_{K}\right)}\left\|\nabla\left(\chi_{R, \varepsilon} \tilde{v}\right)\right\|_{L^{2}\left(G_{K}\right)} .
$$

As $R$ and $\varepsilon$ goes to zero, we have that $\left\|\nabla\left(\chi_{R, \varepsilon} \tilde{v}\right)\right\|_{L^{2}\left(G_{K}\right)}$ goes to zero. We therefore concentrate our attention on the term $\int_{G_{K}} \nabla u \cdot \nabla\left(\left(1-\chi_{R, \varepsilon}\right) \tilde{v}\right)$.

Let $x \in \partial G_{K}$ be such that $x$ does not belong to $\tilde{\gamma} \cup A$. By the convergence in the Hausdorff distance, we can find positive constants $d$ and $d_{1}, n_{0} \in \mathbb{N}$, Lipschitz functions $\varphi: \mathbb{R}^{2} \rightarrow \mathbb{R}$ and, for any $n \geq n_{0}, \varphi_{n}: \mathbb{R}^{2} \rightarrow \mathbb{R}$ such that, setting $D(x)=\left\{y \in \mathbb{R}^{3}: y^{\prime} \in Q_{d}^{\prime}\left(x^{\prime}\right)\right.$ and $\left.x_{3}-d_{1}<y_{3}<\varphi\left(y^{\prime}\right)\right\}$ and $D_{n}(x)=\{y \in$ $\mathbb{R}^{3}: y^{\prime} \in Q_{d}^{\prime}\left(x^{\prime}\right)$ and $\left.x_{3}-d_{1}<y_{3}<\varphi_{n}\left(y^{\prime}\right)\right\}$, the following properties are satisfied for any $n \geq n_{0}$. First, for any $y^{\prime} \in Q_{d}^{\prime}\left(x^{\prime}\right), x_{3}-d_{1}<\varphi\left(y^{\prime}\right), \varphi_{n}\left(y^{\prime}\right)<x_{3}+d_{1}$, $D(x)$ and $D_{n}(x)$ are domains with Lipschitz boundary, and the Lipschitz constants of $\varphi$ and $\varphi_{n}$ are uniformly bounded. Second, up to the same rigid transformation, $\bar{D}(x) \subset \overline{G_{K}} \backslash(\tilde{\gamma} \cup A)$ and $\bar{D}(x) \cap \partial G_{K}=\left\{y \in \mathbb{R}^{3}: y^{\prime} \in \overline{Q^{\prime}}{ }_{d}\left(x^{\prime}\right)\right.$ and $\left.y_{3}=\varphi\left(y^{\prime}\right)\right\}$, and $\bar{D}_{n}(x) \subset \overline{G_{K_{n}}} \backslash(\tilde{\gamma} \cup A)$ and $\bar{D}_{n}(x) \cap \partial G_{K_{n}}=\left\{y \in \mathbb{R}^{3}: y^{\prime} \in \overline{Q^{\prime}}{ }_{d}\left(x^{\prime}\right)\right.$ and $y_{3}=$ $\left.\varphi_{n}\left(y^{\prime}\right)\right\}$. Finally, as $n \rightarrow \infty, D_{n}(x)$ converges to $D(x)$ in the Hausdorff distance.

Let $\hat{v} \in H^{1}(D(x))$ be such that $\hat{v}$ is identically equal to zero on a neighbourhood of $\partial D(x) \backslash \partial G_{K}$. Since $D(x)$ has Lipschitz boundary, we can assume that $\hat{v}$ can be extended in such a way that $\hat{v} \in H_{0}^{1}\left(B_{a}\right)$ for some $a$ sufficiently large and that $\hat{v}$ is identically equal to zero on a neighbourhood of $\partial D_{n}(x) \backslash \partial G_{K_{n}}$ for any $n \geq n_{1}$. Then, we immediately infer that $\int_{D_{n}(x)} \nabla u_{n} \cdot \nabla \hat{v}=0$. Furthermore,

$$
\int_{D_{n}(x)} \nabla u_{n} \cdot \nabla \hat{v}=\int_{D(x)} \nabla u_{n} \cdot \nabla \hat{v}+\int_{D_{n}(x) \backslash D(x)} \nabla u_{n} \cdot \nabla \hat{v}-\int_{D(x) \backslash D_{n}(x)} \nabla u_{n} \cdot \nabla \hat{v} .
$$

Since $\nabla u_{n}$ converges strongly in $L^{2}$ to $\nabla u$ we have that $\int_{D(x)} \nabla u_{n} \cdot \nabla \hat{v} \rightarrow \int_{D(x)} \nabla u$. $\nabla \hat{v}$ as $n \rightarrow \infty$. On the other hand, by a construction similar to the one used before, we can find $q>2$ ad $C>0$ such that for any $n \geq n_{2}$ we have $\left\|\nabla u_{n}\right\|_{L^{q}\left(Q_{d, d_{1}}(x)\right)} \leq$ $C$. Therefore, $\int_{D_{n}(x) \backslash D(x)} \nabla u_{n} \cdot \nabla \hat{v}-\int_{D(x) \backslash D_{n}(x)} \nabla u_{n} \cdot \nabla \hat{v} \rightarrow 0$ as $n \rightarrow \infty$ and we conclude that

$$
\int_{D(x)} \nabla u \cdot \nabla \hat{v}=0
$$

By a localization procedure, for instance by a partition of unity, it is not difficult to conclude that for any $v \in H^{1}\left(G_{K}\right) \cap L^{\infty}\left(G_{K}\right)$ we have $\int_{G_{K}} \nabla u \cdot \nabla\left(\left(1-\chi_{R, \varepsilon}\right) \tilde{v}\right)=0$ 
and, consequently, $\int_{G_{K}} \nabla u \cdot \nabla v=\int_{\tilde{\gamma}} f v$. The result for any $v \in H^{1}\left(G_{K}\right)$ which is not bounded follows by a truncation argument. For any $m \in \mathbb{N}$, let $v_{m}=(v \wedge m) \vee$ $-m=\max \{\min \{u, m\},-m\}$. Then, for any $m$ we have $\int_{G_{K}} \nabla u \cdot \nabla v_{m}=\int_{\tilde{\gamma}} f v_{m}$ and, since, as $m \rightarrow \infty, \int_{G_{K}} \nabla u \cdot \nabla v_{m} \rightarrow \int_{G_{K}} \nabla u \cdot \nabla v$ and $\int_{\tilde{\gamma}} f v_{m} \rightarrow \int_{\tilde{\gamma}} f v$, the proof is concluded.

We conclude the paper by describing some applications of these stability results to inverse problems. Let $f \in L^{p}(\partial \Omega)$ be such that $\operatorname{supp}(f) \subset \tilde{\gamma}$ and $\int_{\partial \Omega} f=0$. Let $K \in \mathcal{C}, u=u(f, K)$ and $g=\left.u\right|_{\gamma}$.

Let us assume that the Cauchy data $(g, f)$ are known up to some error due to noise. Let us fix $\delta>0$, then the noisy Cauchy data are given by $f_{\delta}$ and $g_{\delta}$. Here $f_{\delta}$ belongs to $L^{p}(\partial \Omega)$ and satisfies $\operatorname{supp}\left(f_{\delta}\right) \subset \tilde{\gamma}$ and $\int_{\partial \Omega} f_{\delta}=0$, whereas $g_{\delta}$ belongs

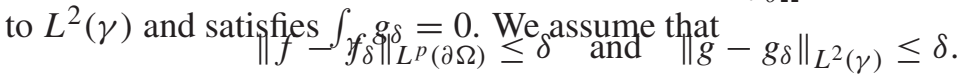

Therefore $\delta$ estimates from above the noise level of the measurements.

The following stability result holds.

Theorem 6.6. For any $\delta>0$, there exists a solution of the following minimization problem

$$
\min _{K \in \mathcal{C}}\left\|\left.u\left(f_{\delta}, K\right)\right|_{\gamma}-g_{\delta}\right\|_{L^{2}(\gamma)} .
$$

If $K_{\delta} \in \mathcal{C}$ is a minimizer and $u_{\delta}=u\left(f_{\delta}, K_{\delta}\right)$ is its corresponding potential, then we have that $u_{\delta}$ converges, as $\delta \rightarrow 0^{+}$, to $u$ weakly* in $B V(\Omega)$ and $\nabla u_{\delta}$ converges to $\nabla u$ in $L^{2}\left(\Omega, \mathbb{R}^{3}\right)$.

Proof. By the direct method in the calculus of variations and Theorem 6.2, we immediately obtain the existence of a minimizer.

Now, let $v_{\delta}=u\left(f_{\delta}, K\right)$. By Proposition 3.1, we have that

$$
\left\|v_{\delta}-g\right\|_{L^{2}(\gamma)} \leq C\left\|f-f_{\delta}\right\|_{L^{p}(\partial \Omega)} \leq C \delta
$$

where $C$ is a positive constant independent of $\delta$. Therefore, we deduce that $\left\|\left.u_{\delta}\right|_{\gamma}-g_{\delta}\right\|_{L^{2}(\gamma)} \leq\left\|v_{\delta}-g_{\delta}\right\|_{L^{2}(\gamma)} \leq\left\|v_{\delta}-g\right\|_{L^{2}(\gamma)}+\left\|g-g_{\delta}\right\|_{L^{2}(\gamma)} \leq(C+1) \delta$.

We conclude that $\left.u_{\delta}\right|_{\gamma}$ converges to $g$ in $L^{2}(\gamma)$ as $\delta \rightarrow 0^{+}$, hence the proof is concluded by using Theorem 6.3 and Theorem 3.3.

\section{References}

[1] G. Alessandrini, E. Beretta, E. Rosset and S. Vessella, Optimal stability for inverse elliptic boundary-value problems with unknown boundaries, Ann. Scuola Norm. Sup. Pisa Cl. Sci. 29 (2000), 755-806.

[2] G. Alessandrini and A. Diaz Valenzuela, Unique determination of multiple cracks by two measurements, SIAM J. Control Optim. 34 (1996), 913-921. 
[3] G. Alessandrini and E. Di Benedetto, Determining 2-dimensional cracks in 3dimensional bodies: uniqueness and stability, Indiana Univ. Math. J. 46 (1997), 1-82.

[4] L. Ambrosio, N. Fusco and D. Pallara, "Functions of Bounded Variation and Free Discontinuity Problems", Clarendon Press, Oxford, 2000.

[5] K. BRYAN and M. S. VogeliUs, A review of selected works on crack identification, In: "Geometric Methods in Inverse Problems and PDE Control", C. B. Croke, I. Lasiecka, G. Uhlmann and M. S. Vogelius (eds.), Springer-Verlag, New York, 2004, 25-46.

[6] G. Dal Maso, F. Ebobisse and M. Ponsiglione, A stability result for nonlinear Neumann problems under boundary variations, J. Math. Pures Appl. 82 (2003), 503-532.

[7] J. DenY and J. L. Lions, Les espaces du type de Beppo Levi, Ann. Inst. Fourier (Grenoble) 5 (1953-54), 305-370.

[8] M. DI CRISTO and L. RONDI, Examples of exponential instability for inverse inclusion and scattering problems, Inverse Problems 19 (2003), 685-701.

[9] L. C. Evans and R. F. Gariepy, "Measure Theory and Fine Properties of Functions", CRC Press, Boca Raton Ann Arbor London, 1992.

[10] H. FedERER, "Geometric Measure Theory", Springer-Verlag, Berlin Heidelberg New York, 1969.

[11] A. Friedman and M. Vogelius, Determining cracks by boundary measurements, Indiana Univ. Math. J. 38 (1989), 527-556.

[12] A. Giacomini, A stability result for Neumann problems in dimension $N \geq 3$, J. Convex Anal. 11 (2004), 41-58.

[13] E. Giusti, "Minimal Surfaces and Functions of Bounded Variation", Birkhäuser, Boston Basel Stuttgart, 1984.

[14] J. Heinonen, T. Kilpeläinen and O. Martio, "Nonlinear Potential Theory of Degenerate Elliptic Equations", Clarendon Press, Oxford New York Tokyo, 1993.

[15] D. S. JERISON and C. E. KenIG, The Neumann problem on Lipschitz domains, Bull. Amer. Math. Soc. (N.S.) 4 (1981), 203-207.

[16] V. G. MAZ'JA, “Sobolev Spaces”, Springer-Verlag, Berlin Heidelberg New York, 1985.

[17] N.G. MEYERS, An $L^{p}$-estimate for the gradient of solutions of second order elliptic divergence equations, Ann. Scuola Norm. Sup. Pisa Cl. Sci. 17 (1963), 189-206.

[18] D. MumFord and J. SHAH, Boundary detection by minimizing functionals, I, In: "Proceedings of IEEE Computer Society Conference on Computer Vision and Pattern Recognition", IEEE Computer Society Press/North-Holland, Silver Spring Md./Amsterdam, 1985, 22-26.

[19] D. MUMFORD and J. SHAH, Optimal approximations by piecewise smooth functions and associated variational problems, Comm. Pure Appl. Math. 42 (1989), 577-685.

[20] M. K. V. MURThy and G. StAmPaCCHIA, A variational inequality with mixed boundary conditions, Israel J. Math. 13 (1972), 188-224.

[21] L. Rond, Uniqueness and Optimal Stability for the Determination of Multiple Defects by Electrostatic Measurements, Ph.D. thesis, S.I.S.S.A.-I.S.A.S., Trieste, 1999 (downloadable from http://www.sissa.it/library/).

[22] L. RonDI, Optimal stability of reconstruction of plane Lipschitz cracks, SIAM J. Math. Anal. 36 (2005), 1282-1292.

[23] L. Rondi and F. SANTOSA, Enhanced Electrical Impedance Tomography via the MumfordShah Functional, ESAIM: COCV 6 (2001), 517-538.

Dipartimento di Matematica e Informatica Università degli Studi di Trieste

Via Valerio, 12/1

34127 Trieste, Italy

rondi@units.it 